VOL. 7 (2003), 107-152

\title{
FUNDAMENTAL FACTS ON TRANSLATIONAL O-REGULARLY VARYING FUNCTIONS
}

\author{
Milan R. Tasković ${ }^{*}$
}

\begin{abstract}
In this paper we introduce three new classes of functions under names translational slowly varying, translational regularly varying and translational $\mathcal{O}$-regularly varying functions. All classes have important applications in the study of asymptotic processes. In this sense, Uniform Convergence Theorem, Characterization Theorem and Representation Theorem are the main results of this paper for all cite classes of functions. This results are closely connected with the Karamata's theory of regularly varying functions. Also, in this paper we introduce three classes of sequences under names translational slowly varying, translational regularly varying and translational $\mathcal{O}$-regularly varying sequences. All three classes have important applications in the study of asymptotic processes. The results are of relevance in connection with limit statements in various branches of probability theory and ergodic theory.
\end{abstract}

\section{Contents}

1. Introduction and history

2. Translational slowly varying functions

3. Translational regularly varying functions

4. Translational $\mathcal{O}$-regularly varying functions

5. Some subclasses of the class $\operatorname{Tr}(O R V)$

6. Translational regularly varying sequences

7. Translational $\mathcal{O}$-regularly varying sequences

8. Some subclasses of the class $\operatorname{Tr}(O R V s)$

9. Some open problems and an illustration

AMS (MOS) Subject Classification 1991. Primary: 26A12, 40A25, 40E05, 46F10. Secondary: 42A20, 43A85, 10K30, 60F05.

Key words and phrases: Translational slowly varying function, Translational regularly varying function, Translational $\mathcal{O}$-regularly varying function, uniform convergence, characterization, representation, Slowly varying function, Regularly varying function, Ergodic Theory, $\mathcal{O}$-regularly varying function, Karamata's theory, translational regularly varying sequences, imbedding sequences in functions, exponential representations of sequences and functions, Karamata's theory of sequences, translational $\mathcal{O}$-regularly varying sequences.

*Research supported by Science Fund of Serbia under Grant 1457. 


\section{Introduction and history}

We shall say that a positive, finite and measurable function $R$, defined on $I_{a}:=[a, \infty)$ for some $a>0$, is a regularly varying function at infinity (denoted this class by $R V$ ) in the sense of Karamata if the limit

$$
\lim _{x \rightarrow \infty} \frac{R(\lambda x)}{R(x)}=k(\lambda)
$$

is positive and finite for each $\lambda>0$. It follows immediately that $k(\lambda)=\lambda^{\rho}$ for some $\rho \in \mathbb{R}$. The number $\rho$ is the index of $R$.

The $R V$ functions of index $\rho=0$ are called slowly varying (denoted this class by $S V$ ) functions and are denoted by $L$. Their interest lies in the fact that $R$ is a $R V$ function of index $\rho$ if and only if $R(x)=x^{\rho} L(x)$ on some $I_{a}$.

Classes $S V$ and $R V$ of slowly and regularly varying functions were introduced by Jovan Karamata in 1930. In this respect we refer to the books of E. Seneta [26] and Bingham-Goldie-Teugels [7]. Both classes have important consequences in the study of asymptotic processes.

In connection with the preceding, the most important properties of $R V$ functions may be stated as follows:

(a) (Characterization Theorem). If $R$ is a regularly varying function, then the limit $k(\lambda)$ in (1) is necessarily of the form $\lambda^{\rho}$ for some $-\infty<\rho<\infty$ and for each $\lambda>0$.

(b) (Uniform Convergence Theorem). The relation (1) holds uniformly for $\lambda$ in any compact interval $I \subset(0, \infty)$.

(c) (Representation Theorem). There exists a number $b \geq a$ such that for $x \geq b$ we have

$$
R(x)=\exp \left(\alpha(x)+\int_{b}^{x} \frac{\beta(t)}{t} d t\right),
$$

where $\alpha$ and $\beta$ are bounded measurable functions on $I_{b}$ such that $\alpha(x)$ converges to a real number and $\beta(x) \rightarrow \rho$ as $x \rightarrow \infty$.

We notice that $R V$ functions have been introduced by J. Karamata [17]. He proved for continuous function $R$ the crucial of the here mentioned results.

The Uniform Convergence Theorem for measurable $S V$ functions was proved by T. van Aardennee-Ehrenfest, N.G. de Bruijn and J. Korevaar [31], H. Delange [13], W. Matuszewska [22], and Bojanić-Seneta [8].

The Representation Theorem for $S V$ funictions $L$ such that $\log L$ is integrable on every compact subinterval of $(a, \infty)$ was proved in [31]. Finally, the Representation Theorem in the present form, for arbitrary measurable $S V$ functions, was established by N. G. de Bruijn [11]; also and Bojanić-Seneta [8]. 
To extend Karamata's ideas to $O$-asymptotic relations in 1936 V. G. Avakumović introduced the class of $\mathcal{O}$-regularly varying functions $K$ (denoted this class by $O R V$ ) which satisfy the condition

$$
0<\liminf _{x \rightarrow \infty} \frac{K(\lambda x)}{K(x)} \leq \limsup _{x \rightarrow \infty} \frac{K(\lambda x)}{K(x)}<\infty
$$

for every $\lambda \geq 1$. In this form $O R V$ functions were introduced by V.G. Avakumović [3] in a note concerning some tauberian theorems, but it was J. Karamata [19] who in 1936 revealed their characteristic properties.

It happened that Bari-Stečkin [5] in their well-known memoir on best approximation, which appeared in 1956, indepedently introduced monotone $O R V$ functions which tend to zero and developed their theory.

J. Karamata in 1936 proved that a $O R V$ function $K$ can be characterized by each of the following conditions:

(a) (Representation Theorem). There exist measurable and bounded real functions $\alpha$ and $\beta$ on $I_{b}$ for some $b \geq a$ such that for $x \geq b$

$$
K(x)=\exp \left(\alpha(x)+\int_{b}^{x} \beta(t) \frac{d t}{t}\right) .
$$

(b) (Characterization Theorem). There exist four numbers $0<m<$ $M<\infty$ and $\rho<\tau$ such that for $y \geq x \geq b$ the following inequalities hold:

$$
m\left(\frac{y}{x}\right)^{\rho} \leq \frac{K(y)}{K(x)} \leq M\left(\frac{y}{x}\right)^{\tau} .
$$

In this paper, we shall introduce some new classes of functions which have further applications in the study of asymptotic processes and ergodic theory. This facts are closely connected with the Karamata's theory of regularly varying functions.

\section{Translational slowly varying functions}

A positive, finite and measurable function $A$, defined on $I_{a}$ for some $a>0$, is said to be translational slowly varying at infinity (denoted this class by $\operatorname{Tr}(S V))$ if the limit

$$
\lim _{x \rightarrow \infty} \frac{A(x+\lambda)}{A(x)}=1
$$

for each $\lambda \geq 0$. The most important properties of $\operatorname{Tr}(S V)$ functions may be stated as follows:

Theorem 2.1. (Uniform Convergence Theorem). If $A$ is a $\operatorname{Tr}(S V)$ function, then for every $[a, b], 0<a<b<\infty$, the relation (2) holds uniformly with respect to $\lambda \in[a, b]$. 
Proof. Let $A$ be a $\operatorname{Tr}(S V)$ function and let $f$ be defined by

$$
f(x)= \begin{cases}0 & \text { if } \quad x<a, \\ \log A(x) & \text { if } \quad x \geq a .\end{cases}
$$

Then, as is easy to see, $f$ is a measurable function on $\mathbb{R}$ and

$$
f(x+\lambda)-f(x) \rightarrow 0 \quad(x \rightarrow \infty)
$$

for every $\lambda \in \mathbb{R}$. If we show that the following fact holds that

$$
\sup _{0 \leq \lambda \leq 1}|f(x+\lambda)-f(x)| \rightarrow 0 \quad(x \rightarrow \infty)
$$

the statement will clearly be proved. Suppose that (3) holds and that (4) is not true. Then we can find $\delta>0$ and sequences $\left(\lambda_{n}\right)$ and $\left(x_{n}\right)$ such that $\lambda_{n} \in[0,1], x_{n} \geq n$, and

$$
\left|f\left(x_{n}+\lambda_{n}\right)-f\left(x_{n}\right)\right| \geq \delta \quad \text { for } n \in \mathbb{N} .
$$

Let $m^{*}$ be the outer measure of subsets of $\mathbb{R}$ and for $0<\varepsilon<\delta / 4$ let

$$
M_{n}:=\left\{t: \sup _{x \geq n}|f(x+t)-f(x)| \leq \varepsilon\right\} .
$$

Since $\left(M_{n} \cap[0,3]\right)$ is an increasing sequence of subsets of $\mathbb{R}$ converging to $[0,3]$ we obtain

$$
\lim _{n \rightarrow \infty} m^{*}\left(M_{n} \cap[0,3]\right)=3,
$$

and hence we can find $s \in \mathbb{N}$ such that $m^{*}\left(M_{s} \cap[0,3]\right) \geq 5 / 2$. Let

$$
\begin{gathered}
B=\left\{t:\left|f(t)-f\left(x_{s}\right)\right| \leq \varepsilon\right\} \cap\left[x_{s}, x_{s}+4\right] \\
C=\left\{t:\left|f(t)-f\left(x_{s}+\lambda_{s}\right)\right| \leq \varepsilon\right\} \cap\left[x_{s}, x_{s}+4\right],
\end{gathered}
$$

then $B$ and $C$ are disjoint measurable subsets of $\left[x_{s}, x_{s}+4\right]$ and thus we have $m(B)+m(C) \leq 4$.

If we denote by $X$ and $Y$ the set $M_{s} \cap[0,3]$ translated by $x_{s}$ and $x_{s}+\lambda_{s}$, respectively, then it is easy to see that $X \subset B$ and $Y \subset C$. Hence, consequently,

$$
\begin{aligned}
& \frac{5}{2} \leq m^{*}\left(M_{s} \cap[0,3]\right)=m^{*}(X) \leq m^{*}(B), \\
& \frac{5}{2} \leq m^{*}\left(M_{s} \cap[0,3]\right)=m^{*}(Y) \leq m^{*}(C),
\end{aligned}
$$

and thus so $m(B)+m(C) \geq 5$, which is impossible in view of the preceding inequality $m(B)+m(C) \leq 4$. Then

For the case of an arbitrary interval $[a, b]$ define $\tilde{f}(x)=f((b-a) x)$.

$$
f(x+\lambda)-f(x)=\tilde{f}(y+\mu)-\tilde{f}(y)+f(x-a)-f(x)
$$


where $y=(x-a) /(b-a), \mu=(\lambda-a) /(b-a)$, so that $y \rightarrow \infty$ if and only if $x \rightarrow \infty$; i.e., $\lambda \in[a, b]$ if and only if $\mu \in[0,1]$. The proof is complete.

Annotation. The preceding proof follows the same ideas as the analogous proof of Lemma 1 in Bojanić - Seneta [8].

The following statement gives an integral representation theorem for functions from the class $\operatorname{Tr}(S V)$.

Theorem 2.2. (Representation Theorem). If $A$ is a $\operatorname{Tr}(S V)$ function, then there exists a positive number $b \geq a$ such that for all $x \geq b$ we have

$$
A(x)=\mu(x) \exp \left(\int_{b}^{x} \varepsilon(t) d t\right),
$$

where $\mu(x)$ is a positive and measurable function on $I_{b}$ such that $\mu(x) \rightarrow c \in$ $(0, \infty)$ as $x \rightarrow \infty$, and $\varepsilon(x)$ is a continuous function on $I_{b}$ such that $\varepsilon(x) \rightarrow 0$ $($ as $x \rightarrow \infty)$. Conversely, if a function $A$ of the form (5), then $A$ is a $\operatorname{Tr}(S V)$ function.

Proof. Let $A$ be a $\operatorname{Tr}(S V)$ function and let $f$ be defined by $f(t)=$ $\log A(t)$. Then, as is easy to see, $f$ is a measurable function for $t \geq b$, where $I_{b}$ is the domain of $A$, and $f$ satisfies the following condition that

$$
f(t+\lambda)-f(t) \rightarrow 0 \quad(t \rightarrow \infty)
$$

uniformly with respect to $\lambda \in[0,1]$. Define the function $f_{1}(t)$ by

$$
f_{1}(t)=f(n)+6[f(n+1)-f(n)] \int_{0}^{t-n} y(1-y) d y
$$

for $n \leq t \leq n+1$, and all $n \in \mathbb{N} \cup\{0\}$. Since

$$
f_{1}^{\prime}(t)=6[f(n+1)-f(n)](t-n)(n+1-t)
$$

for $n \leq t \leq n+1$, it follows that, for all $n \in \mathbb{N} \cup\{0\}, f_{1}^{\prime}(n)=0$. Hence, $f_{1}^{\prime}(t)$ is continuous and

$$
\left|f_{1}^{\prime}(t)\right| \leq \frac{3}{2}|f(n+1)-f(n)|
$$

for $n \leq t \leq n+1$. Also we obtain that

$$
\begin{gathered}
\left|f_{1}(t)-f(t)\right| \leq|f(n)-f(t)|+6|f(n+1)-f(n)|\left|\int_{0}^{t-n} y(1-y) d y\right|= \\
=|f(n)-f(t)|+|f(n+1)-f(n)|(t-n)^{2}(2 n+3-2 t) \leq \\
\leq|f(t)-f(n)|+3|f(n+1)-f(n)|,
\end{gathered}
$$

and thus, as $t \rightarrow \infty$, we have

$$
f_{1}^{\prime}(t) \rightarrow 0 \text { and } f_{1}(t)-f(t) \rightarrow 0 .
$$


Hence, since the function $x \mapsto f_{1}(x)$ it has continuous derivative for $x \geq b$, we obtain

$$
f_{1}(x)=\int_{b}^{x} \varphi(t) d t+\text { constant }
$$

where $x \mapsto \varphi(x)$ is a contunuous function for $x \geq b$. If to differentiate (6) we have

$$
f_{1}^{\prime}(x)=\varphi(x) \text { for } x \geq b,
$$

i.e., from the preceding facts, we obtain

$$
\varphi(x)=\varepsilon(x) \text { for } x \geq b,
$$

with the function $x \mapsto \varepsilon(x)$ which is a continuous function on $I_{b}$ such that $\varepsilon(x) \rightarrow 0$ as $x \rightarrow \infty$. From (6) and (7) we obtain

$$
A_{1}(x)=\exp \left(f_{1}(x)\right)=C \exp \left(\int_{b}^{x} \varepsilon(t) d t\right),
$$

where $C$ is a constant. Also, from the preceding facts, we have

$$
\begin{gathered}
\mu(x)=\frac{A_{1}(x)}{A(x)}=\frac{C \exp \left(f_{1}(x)\right)}{\exp (f(x))}= \\
=C \exp \left(f_{1}(x)-f(x)\right) \rightarrow C \quad(x \rightarrow \infty) .
\end{gathered}
$$

Hence

$$
A(x)=\mu(x) \exp \left(\int_{b}^{x} \varepsilon(t) d t\right),
$$

where $\mu(x)$ and $\varepsilon(t)$ are as required the Representation Theorem.

Conversely, according to these conditions, every the function $A(x)$, with the representation (5), is a measurable function on $I_{b}$ and for every $\lambda \geq 0$ holds

$$
\begin{aligned}
& \left|\frac{A(x+\lambda)}{A(x)}-1\right|=\left|\exp \left(\int_{x}^{x+\lambda} \varepsilon(t) d t\right)-1\right| \leq \\
& \quad \leq \exp \left(\lambda \max _{t \geq x} \varepsilon(t)\right)-1 \rightarrow 0 \quad(x \rightarrow \infty) ;
\end{aligned}
$$

and with this the proof is complete.

Remark. We notice that from the preceding proof of part (8) we have a directly and a simple proof, in the proper manner, of the Theorem 1.

An illustration. We notice that a typical result of the Abelian nature can be stated as follows. Let $k$ be a measurable function such that

$$
\int_{0}^{1} t^{-\delta}|k(t)| d t<\infty \quad \text { and } \quad \int_{1}^{\infty} e^{\delta t}|k(t)| d t<\infty
$$

for some $0<\delta<\infty$, then for every translational slowly varying function $A$ we have

$$
\lim _{x \rightarrow \infty} \int_{0}^{\infty} k(t) \frac{A(x+t)}{A(x)} d t=\int_{0}^{\infty} k(t) d t .
$$




\section{Translational regularly varying functions}

A positive, finite and measurable function $f$, defined on $I_{a}$ for some $a>0$, is said to be translational regularly varying at infinity (denoted this class by $\operatorname{Tr}(R V))$ if the limit

$$
\lim _{x \rightarrow \infty} \frac{f(x+\lambda)}{f(x)}=h(\lambda)
$$

is positive and finite for each $\lambda \geq 0$.

A function $f$ is said to be translational regularly varying at zero if $f(1 / x)$ is translational regularly varying at infinity.

Translational regular variation can now be defined at any finite point a by shifting the origin of the function to this point.

It is thus apparent that it suffices to develop the theory of translation regularly variation at infinity, which we shall do, frequently omitting the words "at infinity" in the sequel.

The fundamental statement of this section is the following, since it shows that $h(\lambda)$ must have the form $e^{\sigma \lambda}$, and so the $f$ considered must be translational regularly varying in the previously defined sense.

Theorem 3.3. (Characterization Theorem). If $f$ is a translational regularly varying function (i.e., $f \in \operatorname{Tr}(R V)$ ), then the limit $h(\lambda)$ in (9) is necessarily of the form $e^{\sigma \lambda}$ for some $-\infty<\sigma<\infty$ and for each $\lambda \geq 0$.

The number $\sigma$ is the index of $f$. The $\operatorname{Tr}(R V)$ functions of index $\sigma=0$ are called translational slowly varying $(\operatorname{Tr}(S V))$ functions and are denoted by $A$. Their interest lies in the fact that $f$ is a $\operatorname{Tr}(R V)$ function of index $\sigma$ if and only if $f(x)=e^{\sigma x} A(x)$ on some $I_{b}$.

We shall proceed by proving Theorem 3 via a well-know variant statement of Cauchy in the following form.

Theorem 3.4. Let $r(y)$ be a real measurable function defined on $I_{b}$ for some $b>0$. If

$$
r(y+\mu)-r(y) \rightarrow \rho(\mu) \quad \text { as } y \rightarrow \infty,
$$

with finite $\rho(\mu)$ and for each $\mu \in \mathbb{R}$, then

$$
\frac{r(y)}{y} \rightarrow \frac{\rho(\mu)}{\mu}=\sigma \quad \text { as } y \rightarrow \infty
$$

for each $\mu \neq 0$. Consequently, then $\rho(\mu)=\sigma \mu$ for $\mu \in \mathbb{R}$. (See: A. Zygmund [34].) 
A brief variant proof of this statement based on Césaro limit of a sequence may be found in E. Seneta [26].

As an immediate application of Theorem 4, as a directly consequence, putting $r(y)=\log f(y), \lambda=\mu$ and $\rho(\mu)=\log h(\mu)$, we obtain the following essential result.

Theorem 3.5. If $f \in \operatorname{Tr}(R V)$, then there exists a real number $\sigma$ such that for every $\lambda \geq 0$ we have that

$$
\frac{f(x+\lambda)}{f(x)} \rightarrow e^{\sigma \lambda} \quad \text { as } x \rightarrow \infty
$$

and such that

$$
\frac{\log f(x)}{x} \rightarrow \sigma \quad \text { as } x \rightarrow \infty .
$$

In connection with preceding facts, in further, from Theorem 5 we have that every translational regularly varying function $f$ has the representation in the form

$$
f(x)=e^{\sigma x} A(x) \text { for } x \geq b,
$$

where $b \geq a$, where $\sigma \in \mathbb{R}$ and $A(x)$ is a translational slowly varying function.

In connection with this, from the preceding section and this facts, we have the following fundamental statement.

Theorem 3.6. (Representation Theorem). A function $f \in \operatorname{Tr}(R V)$ if and only if there exist $\sigma \in \mathbb{R}$ and a positive number $b \geq a$ such that for all $x \geq b$ we have

$$
f(x)=\mu(x) \exp \left(\sigma x+\int_{b}^{x} \varepsilon(t) d t\right),
$$

where $\mu(x)$ is a positive and measurable function on $I_{b}$ such that $\mu(x) \rightarrow c \in$ $(0, \infty)$ as $x \rightarrow \infty$, and $\varepsilon(x)$ is a continuous function on $I_{b}$ such that $\varepsilon(x) \rightarrow 0$ (as $x \rightarrow \infty$ ).

Now, from Theorem 6 , as an immediate consequence, we obtain the following statement on uniformity of convergence in the following sense.

The following statement, the analogue of Theorem 1, ensures, under measurability of $f$, uniformity of convergence of finite intervals in (9).

Theorem 3.7. (Uniform Convergence Theorem). If $f$ is a $\operatorname{Tr}(R V)$ function, then the relation (9) holds uniformly for $\lambda$ in any compact interval $I \subset(0, \infty)$. 
The proof of this statement is analogous to the proof of Theorem 1 .

Annotations. In connection with the Characterization Theorem we notice that from

$$
\frac{f(x+\lambda+\gamma)}{f(x)}=\frac{f(x+\lambda+\gamma)}{f(x+\lambda)} \frac{f(x+\lambda)}{f(x)}
$$

there follows, as $x \rightarrow \infty$,

$$
h(\lambda+\gamma)=h(\gamma) h(\lambda)
$$

for all nonnegative $\lambda$ and $\gamma$.

This is a form of the Cauchy (or Hamel) functional equation on the nonnegative real numbers, for a function $h>0$, which, being a pointwise limit of measurable functions, is measurable.

It is known (see: J. Aczél [1]) that under these conditions the only solutions are of the form $e^{\sigma \lambda}$ for $-\infty<\sigma<\infty$.

Based on the above facts the proof of the preceding statement, as and Theorem 3, we can give also serve as an illustration of the use of Lusin's Theorem in the present setting, which with Egorov's Theorem and Steinhaus's Theorem, appear to be in the natural tools for the present theory.

Examples. The function $A(x)=\log (x+3)$ for $x \geq 0$ belongs to the class $\operatorname{Tr}(S V)$; also, the function

$$
A(x)=\frac{1}{x} \int_{1}^{x} \frac{d t}{1+\log t} \text { for } x \geq 1
$$

belongs to the class of $\operatorname{Tr}(S V)$. On the other hand, the function $f(x)=e^{x}$ for $x \in \mathbb{R}$ belongs to the class $\operatorname{Tr}(R V)$, but $\lim _{x \rightarrow \infty}(f(\lambda x) / f(x))$ does not exist, for example, for $\lambda=3$. Hence $f \notin R V$.

Annotations. We notice, if $f$ is a $\operatorname{Tr}(R V)$ function of index $\sigma$, then, from the preceding facts and results, the following statements hold:

$$
\lim _{x \rightarrow \infty} \frac{\log f(x)}{x}=\sigma .
$$

(b) The function $\log f(x)$ is locally bounded on $I_{b}$ for some $b \geq a$.

(c) $\quad \lim _{x \rightarrow \infty} e^{-\tau x} f(x)=\infty$ for $\tau<\sigma$.

(d) $\quad \lim _{x \rightarrow \infty} e^{-\tau x} f(x)=0$ for $\tau>\sigma$.

(e) For each pair of real numbers $\tau$ and $\rho$ with the property $\tau<\sigma<\rho$, the following facts hold ${ }^{1)}$ :

$$
\begin{aligned}
& \inf _{t \geq x}\left\{e^{-\tau t} f(t)\right\} \sim e^{-\tau x} f(x) \quad \text { as } x \rightarrow \infty, \\
& \sup _{t \geq x}\left\{e^{-\rho t} f(t)\right\} \sim e^{-\rho x} f(x) \quad \text { as } x \rightarrow \infty .
\end{aligned}
$$

${ }^{1)} g(x) \sim \alpha(x)$ means $g(x) / \alpha(x) \rightarrow 1$ as $x \rightarrow \infty$. 
(f) For each pair of real numbers $\tau$ and $\rho$ with the property $\tau<\sigma<\rho$, the following facts hold:

$$
\begin{aligned}
& \sup _{b \leq t \leq x}\left\{e^{-\tau t} f(t)\right\} \sim e^{-\tau x} f(x) \quad \text { as } x \rightarrow \infty, \\
& \inf _{b \leq t \leq x}\left\{e^{-\rho t} f(t)\right\} \sim e^{-\rho x} f(x) \quad \text { as } x \rightarrow \infty .
\end{aligned}
$$

(g) For each $\tau<\sigma$ the following fact holds that is

$$
\lim _{x \rightarrow \infty} \frac{1}{e^{-\tau x} f(x)} \int_{b}^{x} e^{-\tau t} f(t) d t=\frac{1}{\sigma-\tau} .
$$

(h) For each $\tau>\sigma$ the following fact holds that is

$$
\lim _{x \rightarrow \infty} \frac{1}{e^{-\tau x} f(x)} \int_{x}^{\infty} e^{-\tau t} f(t) d t=\frac{1}{\tau-\sigma} .
$$

\section{Translational $\mathcal{O}$-regularly varying functions}

A positive, finite and measurable function $f$, defined on $I_{a}$ for some $a>0$, is said to be translational $\mathcal{O}$-regularly varying at infinity (denoted this class by $\operatorname{Tr}(O R V))$ if the limit

$$
\limsup _{x \rightarrow \infty} \frac{f(x+\lambda)}{f(x)}=r(\lambda)
$$

is finite for each $\lambda \geq 0 .{ }^{2)}$

We notice, from (Eq), there follows, as $x \rightarrow \infty$, that

$$
r(\lambda+\gamma) \leq r(\lambda) r(\gamma)
$$

for all nonnegative $\lambda$ and $\gamma$. Consequently, to this functional inequality, we have that $f$ is a $\operatorname{Tr}(O R V)$ function at infinity if and only if

$$
\left.f(x+\lambda) \asymp f(x) \quad(x \rightarrow \infty) \quad \text { for each } \lambda \geq 0 .{ }^{3}\right)
$$

${ }^{2)}$ Or, equivalently: if

$$
\liminf _{x \rightarrow \infty} \frac{f(x+\lambda)}{f(x)}>0 \text { and } \quad \limsup _{x \rightarrow \infty} \frac{f(x+\lambda)}{f(x)}<\infty
$$

for each $\lambda \geq 0$. (We notice that if $\limsup _{\lambda \rightarrow 0} r(\lambda)=1$, then $\lambda \mapsto r(\lambda)$ is a continuous function.)

${ }^{3)} g(x) \asymp d(x)(x \rightarrow \infty)$ means that there exist two numbers $0<m<M<\infty$ such that the following inequalities hold

$$
m \leq \frac{g(x)}{d(x)} \leq M \quad \text { for } x \text { large enough; }
$$

if we wish to precise that the preceding inequalities hold for $x \geq a$, we write $g(x) \asymp d(x)$ on the interval $I_{a}$. 
Theorem 4.8. If $f$ is a $\operatorname{Tr}(O R V)$ function and I any compact interval in $(0, \infty)$, then

$$
\limsup _{x \rightarrow \infty} \frac{f(x+\lambda)}{f(x)}<\infty
$$

holds uniformly for $\lambda$ in I, i.e.,

$$
\limsup _{x \rightarrow \infty}\left(\sup _{\lambda \in I} \frac{f(x+\lambda)}{f(x)}\right)<\infty .
$$

Corollary 4.9. If I is any compact interval in $(0, \infty)$, then the following fact holds:

$$
\liminf _{x \rightarrow \infty} \frac{f(x+\lambda)}{f(x)}>0 \quad \text { uniformly for } \lambda \text { in } I .
$$

Corollary 4.10. The function $\log r$ defined by (10) is bounded on any compact interval in $(0, \infty)$.

Corollary 4.11. There exists a number $b \geq a$ such that the function $\log f$ is locally bounded on $I_{b}$.

Indeed, let $m, M$ and $b$ be positive real numbers such that the following inequalities hold:

$$
m f(x) \leq f(x+\lambda) \leq M f(x) \text { for each } \lambda \in[1,1+e]
$$

and for each $x \geq b$. Then, any $s \in\left[b e^{n}, b e^{n+1}\right]$, for $n \in \mathbb{N} \cup\{0\}$, is of the form $s=b e^{n}+\lambda$ with $\lambda \in[1,1+e]$, so that

$$
m f\left(b e^{n}\right) \leq f\left(b e^{n}+\lambda\right)=f(s) \leq M f\left(b e^{n}\right) .
$$

Remark. The proof of Theorem 8 follows the same ideas as the proof of Theorem 2.12 in E. Seneta [26] and as the proof of Theorem 1 in Aljančić - Aranđelović [4]. See: Tasković [30].

The following statement, from Theorem 8 , gives an integral representation theorem for functions from the class $\operatorname{Tr}(O R V)$.

Theorem 4.12. (Representation Theorem). A function $f \in \operatorname{Tr}(O R V)$ if and only if there exist $\sigma \in \mathbb{R}$ and measurable and bounded real functions $\mu$ and $\eta$ on $I_{b}$ for some $b \geq a$ such that, for $x \geq b$,

$$
f(x)=\exp \left(\sigma x+\mu(x)+\int_{b}^{x} \eta(t) d t\right) .
$$

We notice that as to conclude, and for the sake of completeness, we give here a proof of the preceding statement, via Theorem 8 , which may be of some interest. 
Proof of Theorem 9. Fix a $b \geq a$ such that (by Corollary 3 of Theorem 8) the function $\log f$ is locally bounded (and so locally integrable) on $I_{b}$. For $s>0$ and $x \geq b$ one has

$$
\begin{gathered}
\int_{0}^{s} \log \frac{f(t+b)}{f(t+x)} d t+\int_{b}^{x} \log \frac{f(t+s)}{f(t)} d t= \\
=\left\{\int_{b}^{b+s}+\int_{x+s}^{x}+\int_{s+b}^{s+x}+\int_{x}^{b}\right\} \log f(u) d u=0 .
\end{gathered}
$$

On the other hand

$$
\int_{0}^{s} \log f(x) d t=s \log f(x),
$$

so that, by addition, we obtain

$$
\int_{0}^{s} \log \left(f(x) \frac{f(t+b)}{f(t+x)}\right) d t+\int_{b}^{x} \log \frac{f(t+s)}{f(t)} d t=s \log f(x) .
$$

Consequently, the function $f$ can be represented in the form (11), where we have, for $\tau \in \mathbb{R}$, that is

$$
\sigma=\frac{\tau}{s}, \quad \eta(x)=\eta_{s}(x)=\frac{1}{s}\left(\log \frac{f(x+s)}{f(x)}-\tau\right)
$$

and

$$
\mu(x)=\mu_{s}(x)=\frac{1}{s}\left\{\int_{0}^{s} \log f(t+b) d t-\int_{0}^{s} \log \frac{f(t+x)}{f(x)} d t\right\}-\frac{b \tau}{s} .
$$

In this sense, the functions $\mu$ and $\eta$ are bounded on $I_{b}$, because first, the function $\log (f(x+t) / f(x))$ is bounded for $0 \leq t \leq s$ and $b \leq x \leq c$ (for each $c \geq b$ ) and second, by Theorem 8, there exists a number $c \geq b$ such that this function is bounded for $0 \leq t \leq s$ and $x \geq c$, too. Hence we have that (10) implies (11).

Conversely, if $f$ is of the form (11) and $\mu$ and $\eta$ are bounded, i.e. $|\mu(x)| \leq$ $M$ and $|\eta(x)| \leq N$ for $x \geq b$, one has for $\lambda \geq 0, x \geq b$ and $x+\lambda \geq b$ we have

$$
\begin{gathered}
\frac{f(x+\lambda)}{f(x)}=\exp \left(\sigma \lambda+\mu(x+\lambda)-\mu(x)+\int_{x}^{x+\lambda} \eta(t) d t\right) \leq \\
\leq \exp (\sigma \lambda+2 M+\lambda N) ;
\end{gathered}
$$

and thus we have that (11) implies (10). The proof is complete.

Theorem 4.13. (Characterization Theorem). A function $f \in \operatorname{Tr}(O R V)$ if and only if at least one of the following conditions hold:

(Ta) There exist four numbers $0<m<M<\infty$ and $\rho<\tau$ such that for all $y \geq x \geq b$ the following inequalities hold:

$$
m \exp (\rho(y-x)) \leq \frac{f(y)}{f(x)} \leq M \exp (\tau(y-x)) .{ }^{4)}
$$


(Tb) There exists a real number $\rho$ such that, as $x \rightarrow \infty$, the following fact holds:

$$
\int_{b}^{x} e^{-\rho t} f(t) d t \asymp e^{-\rho x} f(x) \text { on } I_{c} \text { for each } c>b .
$$

(Tc) There exists a real number $\tau$ such that, as $x \rightarrow \infty$, the following fact holds:

$$
\int_{x}^{\infty} e^{-\tau t} f(t) d t \asymp e^{-\tau x} f(x) \text { on } I_{b} .
$$

In connection with this statement, we notice, moreover, if $f \in \operatorname{Tr}(O R V)$ then, from Theorem 9, we obtain that there exist four numbers $m, M, \rho$ and $\tau$ such that $m=e^{-2 C}, M=e^{2 C}, \rho=-N+\sigma$ and $\tau=N+\sigma$, and where $|\mu(x)| \leq C$ and $|\eta(x)| \leq N$ for $x \geq b$.

Conversely, if (Ta) holds, then directly for $y=x+\lambda \geq x$ as $x \rightarrow \infty$ we obtain that $f$ is a $\operatorname{Tr}(O R V)$ function.

This means that $f \in \operatorname{Tr}(O R V)$ if and only if (Ta). Thus, we need only show that $(\mathrm{Ta})$ is a equivalent to the $(\mathrm{Tb})$ and that $(\mathrm{Tb})$ is a equivalent to the $(\mathrm{Tc})$.

An illustration for the preceding facts is the following case. If $\delta(t)$ is an arbitrary bounded measurable function on the interval $[b,+\infty)$, then for the function $f(x)=\exp \left(\int_{b}^{x} \delta(t) d t\right)$ we have the following inequalities

$$
\exp (\lambda \rho) \leq \frac{f(x+\lambda)}{f(x)} \leq \exp (\lambda \tau)
$$

for all $x \geq b$ and $\lambda \geq 0$, whenever $\rho \leq \delta(t) \leq \tau$ for every $t \geq b$. Hence we have $f \in \operatorname{Tr}(O R V)$.

Second illustration, if $\delta(t)$ is an arbitrary bounded measurable function on the $I_{b}$ for $b \geq a>0$ and $\sigma \in \mathbb{R}$, then for the function of the following form $f(x)=\exp \left(\sigma x+\int_{b}^{x} \delta(t) d t\right)$ we have the following inequalities

$$
\exp ((\sigma+\rho) \lambda) \leq \frac{f(x+\lambda)}{f(x)} \leq \exp ((\sigma+\tau) \lambda)
$$

for all $x \geq b$ and $\lambda \geq 0$, whenever $\rho \leq \delta(t) \leq \tau$ for every $t \geq b$. Hence we obtain $f \in \operatorname{Tr}(O R V)$.

\footnotetext{
${ }^{4)}$ On the other hand, in fact, we notice that instead the fact (Ta) we can supposed that the following fact holds that for a given $\lambda \in(0, \infty)$ there exist $m=m(\lambda)$ and $M=M(\lambda)$ such that

$$
0<m(\lambda) \leq \frac{f(x+t)}{f(x)} \leq M(\lambda)<\infty
$$

for each $t \in[0, \lambda]$. Also, we can supposes a priory that $m$ and $M$ are independent of $\lambda$.
} 
Theorem 4.14. Let $f$ be a $\operatorname{Tr}(O R V)$ function and let $r$ be the positive and finite function on $(0, \infty)$ defined by (10). Then there exist the following limits

$$
p=p(f)=\lim _{t \rightarrow 0+} \frac{\log r(t)}{t} \quad \text { and } \quad q=q(f)=\lim _{t \rightarrow \infty} \frac{\log r(t)}{t} ;
$$

also, then

1) $-\infty<p \leq q<\infty$.

2) $r(t)\left\{\begin{array}{l}\geq e^{q t} \text { for each } t>0 \\ \leq e^{p t} \quad \text { for each } 0<t<1\end{array}\right.$

3) $r(t)=e^{p t}=e^{q t}$ for each $0<t<1$.

Annotation. In connection with this statement, the numbers $p$ and $q$ are the lower index and the upper index of the $\operatorname{Tr}(O R V)$ function $f$. If $p=q$, we say that $f$ is of index $p$. A special interest are $\operatorname{Tr}(O R V)$ functions of index $p=0$; and we call them slow $\operatorname{Tr}(O R V)$ functions. For a given $\operatorname{Tr}(O R V)$ function $f$ define:

$$
\underline{\rho}=\underline{\rho}(f)=\sup _{\eta} \liminf _{x \rightarrow \infty} \eta(x)
$$

and

$$
\bar{\rho}=\bar{\rho}(f)=\inf _{\eta} \limsup _{x \rightarrow \infty} \eta(x),
$$

where the sup and inf are taken over all measurable and bounded functions $\eta$ on $I_{b}$ for which there exists a measurable and bounded function $\mu$ on $I_{b}$ such that (11) holds. In this sense:

$$
\begin{gathered}
\underline{\rho}_{1}=\underline{\rho}_{1}(f)=\sup \left\{\sigma \in \mathbb{R}: e^{-\sigma x} f(x) \text { almost increases }{ }^{5)} \text { on } I_{b}\right\}, \\
\bar{\rho}_{1}=\bar{\rho}_{1}(f)=\inf \left\{\tau \in \mathbb{R}: e^{-\tau x} f(x) \text { almost decreases on } I_{b}\right\}, \\
\underline{\rho}_{2}=\underline{\rho}_{2}(f)=\sup \left\{\sigma \in \mathbb{R}: \int_{b}^{x} e^{-\sigma t} f(t) d t \asymp\right. \\
\left.\asymp e^{-\sigma x} f(x) \text { on } I_{c} \text { for each } c>b\right\}, \\
\bar{\rho}_{2}=\bar{\rho}_{2}(f)=\inf \left\{\tau \in \mathbb{R}: \int_{x}^{\infty} e^{-\tau t} f(t) d t \asymp e^{-\tau x} f(x) \text { on } I_{b}\right\} .
\end{gathered}
$$

\footnotetext{
${ }^{5)} \mathrm{A}$ function $f$ positive and finite on $I_{b}$ is said to be almost increasing on $I_{c}(c \geq b)$ for $x \geq c$ if there exists a constant $M \geq 1$ such that

$$
f(x) \leq M f(y) \text { for each } y \geq x \geq c,
$$
}

or equivalently, if

$$
f(x) \leq M \inf _{t \geq x} f(t) \quad \text { for each } x \geq c .
$$


Suppose, in the sequel, that $f$ is a $\operatorname{Tr}(O R V)$ function of lower and upper index $p$ and $q$, respectively.

Annotation. We notice, in connection with the preceding facts, that if $f$ is a measurable and almost increasing function on $I_{c}$, then:

$$
\begin{aligned}
& F_{\alpha}(x):=\int_{c}^{x} e^{-\alpha t} f(t) d t \preccurlyeq e^{-\alpha x} f(x) \quad \text { on } I_{c} \text { for each } \alpha<0, \\
& F_{\alpha}^{*}(x):=\int_{x}^{\infty} e^{-\alpha t} f(t) d t \succcurlyeq e^{-\alpha x} f(x) \quad \text { on } I_{c} \text { for each } \alpha>0,
\end{aligned}
$$

for which $F_{\alpha}^{*}(c)$ is finite. Indeed, from $f(t) \leq M f(x)$ for $c \leq t \leq x$, there follows

$$
F_{\alpha}(x) \leq M f(x) \int_{c}^{x} e^{-\alpha t} d t \leq M f(x) \int_{0}^{x} e^{-\alpha t} d t \leq-\frac{M}{\alpha} e^{-\alpha x} f(x),
$$

and similarly, $M f(t) \geq f(x)$ for $t \geq x \geq c$ implies that

$$
M F_{\alpha}^{*}(x) \geq f(x) \int_{x}^{\infty} e^{-\alpha t} d t=\frac{1}{\alpha} e^{-\alpha x} f(x) .
$$

On the other hand, if $f$ is a measurable and almost decreasing function

A function $f(x)$ is said to be almost increasing when $x \rightarrow \infty$ (i.e., for $x$ large enough) if it is almost increasing on some interval $I_{c}$, or, equivalently, if

$$
f(x) \preccurlyeq \inf _{t \geq x} f(t) \quad \text { as } x \rightarrow \infty .
$$

In this sense, we note that the last relation has the same meaning as

$$
f(x) \asymp \inf _{t \geq x} f(t) \quad \text { as } x \rightarrow \infty .
$$

Also, by duality (with respect to the ordered set of positive real numbers in which $f$ takes his values), a function $f$ is said to be almost decreasing on $I_{c}$ for $x \geq c$ if there is a constant $0<m \leq 1$ such that

$$
f(x) \geq m f(y) \quad \text { for each } y \geq x \geq c,
$$

or, equivalently,

$$
f(x) \geq m \sup _{t \geq x} f(t) \quad \text { for each } x \geq c ;
$$

similarly, a function $f(x)$ is said to be almost decreasing when $x \rightarrow \infty$ if

$$
f(x) \succcurlyeq \sup _{t \geq x} f(t) \quad(x \rightarrow \infty),
$$

or, equivalently, if

$$
f(x) \asymp \sup _{t \geq x} f(t) \quad(x \rightarrow \infty) .
$$


on $I_{c}$, then:

$$
\begin{gathered}
F_{\alpha}^{*}(x) \preccurlyeq e^{-\alpha x} f(x) \text { on } I_{c} \text { for each } \alpha>0, \\
F_{\alpha}(x) \succcurlyeq e^{-\alpha x} f(x) \quad \text { on } I_{c^{\prime}} \text { for each } \alpha<0 \text { and each } c^{\prime}>c .
\end{gathered}
$$

The proof for this is very similar with the preceding proof of (17) and (18). Indeed, from $m f(t) \leq f(x)$ for $t \geq x \geq c$ there follows:

$$
m F_{\alpha}^{*}(x)=m \int_{x}^{\infty} e^{-\alpha t} f(t) d t \leq f(x) \int_{x}^{\infty} e^{-\alpha t} d t=\frac{1}{\alpha} e^{-\alpha x} f(x)
$$

for $x \geq c$; and $f(t) \geq m f(x)$ for $x \geq t \geq c$ implies

$$
F_{\alpha}(x)=\int_{c}^{x} e^{-\alpha t} f(t) d t \geq m f(x) \int_{c}^{x} e^{-\alpha t} d t \geq \frac{m}{-\alpha}\left(1-e^{-\alpha c}\right) e^{-\alpha x} f(x) .
$$

In further, we notice that, the proof of Theorem 11 is based on the following statement and its consequences.

Lemma 1. Let the function $\rho$ be positive, finite and locally bounded from above on $(0, \infty)$ and such that $\rho(s+t) \leq \rho(s) \rho(t)$ for each $s$ and $t$. Then

$$
\lim _{t \rightarrow \infty} \frac{\log \rho(t)}{t}=\inf _{t>0} \frac{\log \rho(t)}{t} .
$$

Proof. For proof of the fact (21) it is enough to prove that the following inequality holds

$$
\limsup _{t \rightarrow \infty} \frac{\log \rho(t)}{t} \leq \frac{\log \rho(t)}{t} \text { for each } t>0 ;
$$

in this sense, fix a $t>0$ and let $s=t$. Then for $n \in \mathbb{N}$ we have first

$$
\rho(n t) \leq(\rho(t))^{n} \text { for } t>0,
$$

and, dividing by $n t>0$, we obtain

$$
\frac{\log \rho(n t)}{n t} \leq \frac{\log (\rho(t))^{n}}{n t}=\frac{\log \rho(t)}{t} ;
$$

hence, for $x \geq k t(k=1,2, \ldots)$, there exists some integer $m \geq k$ such that $m t \leq x<(m+1) t$. By the preceding inequality

$$
\sup _{x \geq k t} \frac{\log \rho(x)}{x}=\sup _{n \geq k} \sup _{n t \leq u<(n+1) t} \frac{\log \rho(u)}{u} \leq \sup _{n \geq k} \frac{\log \rho(t)}{t}=\frac{\log \rho(t)}{t}
$$

and the required statement follows by letting $k \rightarrow \infty$. The proof is compleete.

Corollary 4.15. Let the function $\rho$ be positive, finite and locally bounded from above on $(0,1]$ and such that $\rho(s+t) \leq \rho(s) \rho(t)$ for each $s$ and $t$ in $(0,1]$. Then

$$
\lim _{t \rightarrow 0+} \frac{\log \rho(t)}{t}=\sup _{0<t<1} \frac{\log \rho(t)}{t} .
$$


We notice that for the proof of this statement one has only to apply Lemma 1 to the function $\rho(1 / t)$ for $t>0$.

Corollary 4.16. Let the function $\rho$ be positive, finite and locally bounded from above on $(0, \infty)$ and such that $\rho(s+t) \leq \rho(s) \rho(t)$ for all $s, t \geq 0$. Denote by $q$ and $p$ the limits in (21) and (22), respectively, then

$$
-\infty<p \leq q<\infty \text {. }
$$

Proof. Facts $q<\infty$ and $p>-\infty$ follow from (21) and (22), respectively. From $\rho(s+t) \leq \rho(s) \rho(t)$ it follows that $(\rho(1 / t))^{1 / t} \leq(\rho(t))^{t}$ for $t$ large enough, i.e., we obtain

$$
\frac{\log \rho(1 / t)}{1 / t} \leq \frac{\log \rho(t)}{t}
$$

and hence the statement $p \leq q$ directly follows by leetting $t \rightarrow \infty$. The proof is complete.

Corollary 4.17. Let the function $\rho$ be positive, finite and locally bounded from above on $(0,+\infty)$ and such that $\rho(s+t) \leq \rho(s) \rho(t)$ for all $s, t \geq 0$, then

$$
\rho(t) \begin{cases}\geq e^{q t} & \text { for each } t \geq 0 \\ \leq e^{p t} & \text { for each } 0<t<1,\end{cases}
$$

where $q$ and $p$ denoted the limits in (21) and (22), respectively.

Proof of Theorem 11. The proof of this statement is now immediate, since the function $r$ of Theorem 11 satisfies all hypotheses required for the function $\rho$ in Lemma 1 and its Corollaries (definition (10) and its immediate consequence and Corollary 2 of Theorem 8).

Lemma 2. Let $f$ be of the form (11). Then the function $e^{-\lambda x} f(x)$ almost increases on $I_{b}$ for $\lambda<\sigma+\liminf _{x \rightarrow \infty} \eta(x)$ and almost decreases on $I_{b}$ for $\lambda>\sigma+\lim \sup _{x \rightarrow \infty} \eta(x)$.

We notice, using the numbers defined and denoted by $\bar{\rho}, \underline{\rho},(13)$ and (14), the result of Lemma 2 may be stated as follows:

$$
\underline{\rho}(f) \leq \underline{\rho}_{1}(f) \text { and } \bar{\rho}(f) \geq \bar{\rho}_{1}(f) .
$$

Proof of Lemma 2. From (11) follows

$$
\frac{e^{-\alpha y} f(y)}{e^{-\alpha x} f(x)}=\exp \left(\mu(y)-\mu(x)+\int_{x}^{y}(\eta(t)+\sigma-\alpha) d t\right)
$$

for $y \geq x \geq b$ and for any real number $\alpha$.

The function $\exp \mu(x)$ being bounded away from both 0 and $\infty$, and so almost increasing and almost decreasing on $I_{b}$, we can assume that $\mu=0$. For the same reason the function $f$ is almost increasing and almost decreasing on 
every finite interval $[b, c]$ for $c \geq b$. Hence, it is sufficient to prove that Lemma 1 holds on some interval $I_{c}(c \geq b)$. Choose $c \geq b$ such that $\eta(t) \geq \sigma-\lambda$ $(\leq \sigma-\lambda)$ for $t \geq c$. From (25) with $\alpha=\lambda$ then follows

$$
\frac{e^{-\lambda y} f(y)}{e^{-\lambda x} f(x)}=\exp \left(\int_{x}^{y}(\eta(t)+\sigma-\lambda) d t\right) \geq e^{0}=1 \quad\left(\leq e^{0}=1\right)
$$

for $y \geq x \geq c$. The proof is complete.

Lemma 3. Let $f$ be a positive and measurable function on $I_{b}$. Suppose that there exist two numbers $\sigma_{0}$ and $\tau_{0}$ such that $e^{-\sigma_{0} x} f(x)$ almost increases and that $e^{-\tau_{0} x} f(x)$ almost decreases on $I_{b}$. Then

$$
\int_{b}^{x} e^{-\rho t} f(t) d t \asymp e^{-\rho x} f(x) \quad \text { on } I_{c}
$$

for each $c>b$ and each $\rho<\sigma_{0}$, and

$$
\int_{x}^{\infty} e^{-\tau t} f(t) d t \asymp e^{-\tau x} f(x) \quad \text { on } I_{b}
$$

for each $\tau>\tau_{0}$.

Proof. The statement follows immediately from the properties (17), (18), (19) and (20) of almost monotone functions: one obtains (26) by putting $f(t)$ with $e^{-\sigma_{0}} f(t)$ and $\alpha=\rho-\sigma_{0}$ in (17) and (20); and, similarly, (27) follows by putting $f(t)$ with $e^{-\tau_{0} t} f(t)$ and $\alpha=\tau-\tau_{0}$ in (18) and (19). The proof is complete.

We notice, using the numbers defined by (13), (14), (15) and (16), the result of Lemma 3 can be stated as follows:

$$
\underline{\rho}_{1}(f) \leq \underline{\rho}_{2}(f) \quad \text { and } \quad \bar{\rho}_{2}(f) \leq \bar{\rho}_{1}(f) .
$$

The previous reasoning and Lemma 3 show that the sets in (15) and (16) are intervals.

Lemma 4. Let $f$ be a positive, measurable and locally bounded function on $I_{b}$. Suppose that for a real number $\rho$ the fact (26) holds for some $c>b$. Then $f$ can be written in the form (11), where $\mu$ and $\eta$ are measurable bounded functions on $I_{b}$ and

$$
\liminf _{x \rightarrow \infty} \eta(x)>\rho-\sigma .
$$

Proof. For $b \leq x<c$ we can take $\mu(x)=\log f(x)$ and $\eta(x)=0$. Hence, it is enough to determine $\mu$ and $\eta$ for $x \geq c$. Let $m$ and $M$ be real numbers such that $0<m \leq 1 \leq M$ and

$$
m \leq B(x)=\frac{e^{-\rho x} f(x)}{\int_{b}^{x} e^{-\rho t} f(t) d t} \leq M \quad \text { for } x \geq c .
$$


On the other hand, since

$$
\int_{c}^{y} B(x) d x=\left[\log \int_{b}^{x} e^{-\rho t} f(t) d t\right]_{c}^{y}
$$

for $y \geq c$, and so for $x \geq c$

$$
\begin{gathered}
f(x)=e^{\rho x} B(x) \int_{b}^{x} e^{-\rho t} f(t) d t= \\
=\left\{e^{\rho c} B(x) \int_{b}^{c} e^{-\rho t} f(t) d t\right\}\left\{e^{\rho(x-c)} \exp \left(\int_{c}^{x} B(t) d t\right)\right\} ;
\end{gathered}
$$

hence statement follows if we put

$$
\mu(x)=\log \left\{e^{(\rho-\sigma) c} B(x) \int_{b}^{c} e^{-\rho t} f(t) d t\right\} \quad \text { and } \quad \eta(x)=B(x)+\rho-\sigma .
$$

Lemma 5. Let $f$ be a positive, measurable and locally bounded function on $I_{b}$. Suppose that for a real number $\tau$ the fact (27) holds. Then $f$ can be written in the form (11), where $\mu$ and $\eta$ are measurable bounded functions on $I_{b}$ and

$$
\limsup _{x \rightarrow \infty} \eta(x)<\tau-\sigma .
$$

Proof. Let $m$ and $M$ be real numbers such that $0<m \leq 1 \leq M$ and

$$
m \leq G(x)=\frac{e^{-\tau x} f(x)}{\int_{x}^{\infty} e^{-\tau t} f(t) d t} \leq M
$$

for $x \geq b$. Since

$$
\int_{b}^{y} G(t) d t=-\left[\log \int_{x}^{\infty} e^{-\tau t} f(t) d t\right]_{b}^{y}=\log \frac{\int_{b}^{\infty} e^{-\tau t} f(t) d t}{\int_{y}^{\infty} e^{-\tau t} f(t) d t}
$$

for $y \geq b$, and so for $x \geq b$

$$
\begin{gathered}
f(x)=e^{\tau x} G(x) \int_{x}^{\infty} e^{-\tau t} f(t) d t= \\
=\left\{e^{\tau b} G(x) \int_{b}^{\infty} e^{-\tau t} f(t) d t\right\}\left\{e^{\tau(x-b)} \exp \left(\int_{b}^{x}(-G(t)) d t\right)\right\}
\end{gathered}
$$

hence statement follows if we put

$$
\mu(x)=\log \left\{e^{(\tau-\sigma) b} G(x) \int_{b}^{\infty} e^{-\tau t} f(t) d t\right\} \quad \text { and } \quad \eta(x)=\tau-\sigma-G(x) .
$$

We notice, using the numbers defined by $(\eta \mathrm{s}),(\eta \mathrm{i}),(15)$ and $(16)$ the result of Lemmas 4 and 5 can be stated as follows:

$$
\underline{\rho}_{2}(f) \leq \underline{\rho}(f) \quad \text { and } \quad \bar{\rho}(f) \leq \bar{\rho}_{2}(f) .
$$


Moreover, the realations (24), (28) and (29) imply the following equalities:

$$
\underline{\rho}(f)=\underline{\rho}_{1}(f)=\underline{\rho}_{2}(f) \text { and } \bar{\rho}(f)=\bar{\rho}_{1}(f)=\bar{\rho}_{2}(f) .
$$

From this facts we have as an immediately consequence a part of the following statement.

Theorem 4.18. Let $f$ be a $\operatorname{Tr}(O R V)$ function of lower and upper index $p$ and $q$, respectively. Then

$$
p=\underline{\rho}=\underline{\rho}_{1}=\underline{\rho}_{2} \quad \text { and } \quad q=\bar{\rho}=\bar{\rho}_{1}=\bar{\rho}_{2} .
$$

We notice, from (30), for proof of Theorem 12, i.e., (31), we need only show that $p=\underline{\rho}$ and $q=\bar{\rho}$. On this sense the following statement holds.

Lemma 6. Let $f$ be a $\operatorname{Tr}(O R V)$ function of lower and upper index $p$ and $q$ respectively. Then

$$
p=\underline{\rho}_{1}(f) \quad \text { and } \quad q=\bar{\rho}_{1}(f) .
$$

Proof. We shall prove the second relation in (32). By applying it to the function $1 / f$ one obtains the first one.

If $e^{-\tau x} f(x)$ almost decreases for $x \geq b$, there exists by definition a real number $M \geq 1$ such that

$$
\frac{e^{-\tau y} f(y)}{e^{-\tau x} f(x)} \leq M \quad \text { for } y \geq x \geq b .
$$

In further, by putting $y=x+t$, this inequality becomes

$$
\frac{f(x+t)}{f(x)} \leq M e^{\tau t} \text { for } x \geq b \text { and } t \geq 0,
$$

and hence

$$
r(t)=\limsup _{x \rightarrow \infty} \frac{f(x+t)}{f(x)} \leq M e^{\tau t} \quad \text { for } t \geq 0 ;
$$

and by 2 ) of Theorem 11 we obtain

$$
e^{q t} \leq M^{\tau t} \text { fot } t>0
$$

i.e., consequently of this, $q \leq \tau$. If $\tau>q$, then, by definition (12) of the upper index $q$, there exists a number $d>1$ such that

$$
\frac{\log r(d)}{d}<\tau, \text { i.e., } r(d)<e^{\tau d} .
$$

Consequently of this, by definition of the function $r$, there exists $x_{0} \geq b$ such that

$$
\frac{f(x+d)}{f(x)}<e^{\tau d} \text { for } x \geq x_{0} .
$$


On the other hand, by Theorem 8 , there exist real numbers $M>0$ and $x_{1} \geq b$ such that

$$
\frac{f(x+s)}{f(x)} \leq M \quad \text { for } x \geq x_{1} \text { and } 1 \leq s \leq d
$$

then, for $c=\max \left\{x_{0}, x_{1}\right\}$, for $x \geq c$ and for some $n \in \mathbb{N} \cup\{0\}$ if $t=s+n d$ we have

$$
\begin{gathered}
\frac{f(x+t)}{f(x)}=\frac{f(x+s+n d)}{f(x+n d)} \frac{f(x+n d)}{f(x+(n-1) d)} \ldots \frac{f(x+d)}{f(x)} \leq \\
\leq M\left(e^{\tau d}\right)^{n}=M e^{\tau n d}=M e^{\tau(t-s)} \leq M e^{\tau t} .
\end{gathered}
$$

Hence, the function $e^{-\tau x} f(x)$ is almost decreasing for $x \geq c$. Being logarithmically bounded on $[b, c]$, the same is true for $b \leq x \leq c$ and, hence, for $x \geq b$. The proof is complete.

Proof of Theorem 10. Directly, from Lemmas 2, 3, 4 and 5 follows equivalence of conditions ( $\mathrm{Ta}),(\mathrm{Tb})$ and $(\mathrm{Tc})$ by the facts: (Ta) implies $(\mathrm{Tb})$ and (Tc) - from Lemma 3, Lemma 4 implies that $f \in \operatorname{Tr}(O R V)$ is a consequenca of $(\mathrm{Tb})$, Lemma 2 implies that (Ta) is a consequence of $f \in \operatorname{Tr}(O R V)$, and Lemma 5 implies that $f \in \operatorname{Tr}(O R V)$ is a consequenca of (Tc). The proof is complete.

Theorem 4.19. Let $f$ be a $\operatorname{Tr}(O R V)$ function of lower and upper index $p$ and $q$, respectively. Then

$$
\begin{array}{cl}
\lim _{x \rightarrow \infty} e^{-\sigma x} f(x)=\infty & \text { for each } \sigma<p, \\
\lim _{x \rightarrow \infty} e^{-\tau x} f(x)=0 & \text { for each } \tau>q .
\end{array}
$$

Annotation. For the proof of this preceding statement we have to introduce the following numbers:

$$
\rho_{*}=\rho_{*}(f)=\liminf _{x \rightarrow \infty} \frac{\log f(x)}{x} \quad \text { and } \quad \rho^{*}=\rho^{*}(f)=\limsup _{x \rightarrow \infty} \frac{\log f(x)}{x}
$$

for the $\operatorname{Tr}(O R V)$ function $f$, and so, we proved that

$$
\rho_{*}(f)=\sup \left\{\sigma \in \mathbb{R}: e^{\sigma x} \preccurlyeq f(x) \text { as } \quad x \rightarrow \infty\right\} .
$$

In the equality (36) one can, evidently, substitute $\preccurlyeq$ by $\succcurlyeq$, i.e.,

$$
\liminf _{x \rightarrow \infty} e^{-\tau x} f(x)>0
$$

by $\lim _{x \rightarrow \infty} e^{-\sigma x} f(x)=\infty$. Since $\rho^{*}(f)=-\rho_{*}(1 / f)$, we have

$$
\rho^{*}=\rho^{*}(f)=\inf \left\{\tau \in \mathbb{R}: f(x) \preccurlyeq e^{\tau x} \quad \text { as } \quad x \rightarrow \infty\right\},
$$

where $f(x) \preccurlyeq e^{\tau x}$ can be substituted by $f(x) \succcurlyeq e^{\tau x}$, i.e., $\limsup _{x \rightarrow \infty} e^{-\tau x} f(x)<\infty$ by $\lim _{x \rightarrow \infty} e^{-\tau x} f(x)=0$. (For further facts see: Tasković [30]). 
Theorem 4.20. Let $f$ be a $\operatorname{Tr}(O R V)$ function of lower and upper index $p$ and $q$, respectively. Then, if $\sigma<p$ the following equivalent realtions are true:

(40) there exists a positive nondecreasing function $\psi$ on $I_{b}$ such that

$$
f(x) \asymp e^{\sigma x} \psi(x) \text { on } I_{b} ;
$$

if $\tau>q$ the following realtions are true:

$$
\begin{aligned}
& \sup _{t \geq x} e^{-\tau t} f(t) \asymp e^{-\tau x} f(x) \quad \text { on } I_{b}, \\
& \inf _{b \leq t \leq x} e^{-\tau t} f(t) \asymp e^{-\tau x} f(x) \text { on } I_{b},
\end{aligned}
$$

there exists a positive nonincreasing function $\psi$ on $I_{b}$ such that

$$
f(x) \asymp e^{\tau x} \psi(x) \text { on } I_{b} .
$$

Proof. If $\sigma<p$, then the function $e^{-\sigma x} f(x)$ almost increases on $I_{b}$, and by the definition and some properties of almost monotone functions ${ }^{6)}$ this is equivalent to each of the facts (38), (39) and (40). The dual statements (41), (42) and (43) follows in analogous way. The proof is complete.

An important remark. The proofs of Theorems 8-14 I am make by the pattern from papers: Aljančić-Aranđelović [4], Matuszewska [22], Matuszewska-Orlicz [23], and Karamata [19].

\section{Some subclasses of the class $\operatorname{Tr}(O R V)$}

From the preceding facts we have the following relations: $\operatorname{Tr}(S V) \subset$ $\operatorname{Tr}(R V) \subset \operatorname{Tr}(O R V)$. In this sense we give a number new examples of subclasses functions of the class $\operatorname{Tr}(O R V)$.

\footnotetext{
${ }^{6)} \mathrm{A}$ function $f$ is almost increasing on $I_{c}$ if and only if there exists an increasing function $\psi$ on $I_{c}$ such that $f(x) \asymp \psi(x)$ on $I_{c}$.

If $\log f$ is locally bounded on $I_{c}$ (bounded on $[c, d]$ for each $d \geq c$ ), then the following facts are equivalent:

$$
\begin{aligned}
& f \text { is almost increasing on } I_{c} . \\
& \sup _{c \leq t \leq x} f(t) \preccurlyeq f(x) \quad \text { as } x \rightarrow \infty . \\
& \sup _{c \leq t \leq x} f(t) \asymp f(x) \quad \text { as } x \rightarrow \infty .
\end{aligned}
$$

In this sense dual facts holds and for almost decreasing functions. This follows in an analogous way.
} 
Example 1. $(\operatorname{Tr}(E R V)$ functions $)$. A positive, finite and measurable function $f$, defined on $I_{a}$ for some $a>0$, is said to be translational extended regular variation at infinity (denoted this class by $\operatorname{Tr}(E R V)$ ) if

$$
\exp (\lambda d) \leq \liminf _{x \rightarrow \infty} \frac{f(x+\lambda)}{f(x)} \leq \limsup _{x \rightarrow \infty} \frac{f(x+\lambda)}{f(x)} \leq \exp (\lambda \rho)
$$

for some $d, \rho \in \mathbb{R}$ and for every $\lambda \geq 0$. We have clearly $\operatorname{Tr}(R V) \subset \operatorname{Tr}(E R V) \subset$ $\operatorname{Tr}(O R V)$, i.e., $\operatorname{Tr}(E R V)$ is a subclass of the class of all $\operatorname{Tr}(O R V)$ functions.

Annotation. We notice, an intermediary class of functions of extended regular variation (denoted by $E R V$ ) was found by Matuszewska - Orlicz [23]. In this sense, a positive, finite and measurable function $f \mid I_{a}$ is said to be $E R V$ if

$$
\lambda^{d} \leq \liminf _{x \rightarrow \infty} \frac{f(\lambda x)}{f(x)} \leq \limsup _{x \rightarrow \infty} \frac{f(\lambda x)}{f(x)} \leq \lambda^{\rho}
$$

for some $d, \rho \in \mathbb{R}$ and for every $\lambda \geq 1$. As well-known $S V \subset R V \subset E R V \subset$ $O R V$. For more detail see [7].

Example 2. $\left(\operatorname{Tr}\left(S_{c}\right)\right.$ functions $)$. A positive finite and measurable function $f \mid I_{a}$ is said to be translational $S_{c}$ varying at infinity (denoted this class by $\left.\operatorname{Tr}\left(S_{c}\right)\right)$ if the limit

$$
\lim _{x \rightarrow \infty} \frac{f(x+\psi(x))}{f(x)}=1
$$

for every continuous function $\psi: I_{a} \rightarrow(0, \infty)$ tending to 0 as $x \rightarrow \infty$. That is $\operatorname{Tr}\left(S_{c}\right) \subset \operatorname{Tr}(O R V)$ follows from the following statement.

Lemma 7. A function $f \in \operatorname{Tr}\left(S_{c}\right)$ if and only if the following fact holds that is

$$
\lim _{\substack{x \rightarrow \infty \\ \lambda \rightarrow 0}} \frac{f(x+\lambda)}{f(x)}=1 .
$$

Proof. If (44) is not true, then we can find sequences $\left(\lambda_{n}\right)$ and $\left(x_{n}\right)$ such that $\lambda_{n} \rightarrow 0$ and $x_{n} \rightarrow \infty$ as $n \rightarrow \infty$, and

$$
\left|\frac{f\left(x_{n}+\lambda_{n}\right)}{f\left(x_{n}\right)}-1\right| \geq \varepsilon \quad \text { for } n \in \mathbb{N} .
$$

If define $\psi\left(x_{n}\right)=\lambda_{n}$ for $n \in \mathbb{N}$ and elsewhere to be linear and continuous, then, clearly, $\psi(x) \rightarrow 0$ as $x \rightarrow \infty$ and

$$
\left|\frac{f\left(x_{n}+\psi\left(x_{n}\right)\right)}{f\left(x_{n}\right)}-1\right|=\left|\frac{f\left(x_{n}+\lambda_{n}\right)}{f\left(x_{n}\right)}-1\right| \geq \varepsilon
$$

for $n \in \mathbb{N}$. Hence $f \notin \operatorname{Tr}\left(S_{c}\right)$. (For the second proof of this see: Tasković [30].) 
Conversely, assume that $f$ is satisfying the condition (44). Then for any $\varepsilon>0$ there are $\delta_{0}(\varepsilon)$ and $\gamma(\varepsilon)$ such that $|(f(x+\lambda) / f(x))-1|<\varepsilon$ if $|\lambda| \leq \delta_{0}(\varepsilon)$ and $x \geq \gamma(\varepsilon)$. If $\psi(x) \rightarrow 0$ as $x \rightarrow \infty$, then there is a $x_{0}$ such that $|\psi(x)| \leq \delta_{0}(\varepsilon)$ for $x \geq x_{0}$. Thus, $|(f(x+\psi(x)) / f(x))-1|<\varepsilon$ for $x \geq \max \left\{x_{0}, \gamma(\varepsilon)\right\}$, which means that $f \in \operatorname{Tr}\left(S_{c}\right)$. The proof is complete.

Annotation. A function $f$ is a $\varphi$-function if $f:[0, \infty) \rightarrow[0, \infty), f(0)=$ $0, f$ is continuous and increasing, and $f(x) \rightarrow \infty$ as $x \rightarrow \infty$.

From Matuszewska - Orlicz [23], also see and Schmidt [25], $K_{c}$ is the set of all $\varphi$-functions $f$ with the property

$$
\lim _{x \rightarrow \infty} \frac{f(\alpha(x) x)}{f(x)}=1
$$

for every continuous function $\alpha:[0, \infty) \rightarrow(0, \infty)$ tending to 1 as $x \rightarrow \infty$. From D. Đurčić [32] follows that $f \in K_{c}$ if and only if

$$
\lim _{\substack{x \rightarrow \infty \\ \lambda \rightarrow 1}} \frac{f(\lambda x)}{f(x)}=1 .
$$

Example 3. $(\operatorname{Tr}(V D)$ functions). Consider arbitrary functions $x$ and $y$ which are positive on the interval $I_{a}$ for some $a>0$ and $x(t), y(t) \rightarrow \infty$ as $t \rightarrow \infty$. If in addition $f$ is positive and measurable on $I_{a}$ we can consider the following fact:

$$
\lim _{t \rightarrow \infty}(x(t)-y(t))=0 \quad \text { implies } \quad \lim _{t \rightarrow \infty} \frac{f(x(t))}{f(y(t))}=1 .
$$

The class of all functions $f$ which satisfying the condition (45) is denoted by $\operatorname{Tr}(V D)$.

Lemma 8. For a positive and measurable function $f: I_{a} \rightarrow(0, \infty)$ the following facts are mutually equivalent:

(a) $f \in \operatorname{Tr}(V D)$.

(b) If $\left(a_{n}\right)$ and $\left(b_{n}\right)$ are arbitrary sequences tending to $\infty$, then we have that $\lim _{n \rightarrow \infty}\left(a_{n}-b_{n}\right)=0$ implies that $\lim _{n \rightarrow \infty}\left(f\left(a_{n}\right) / f\left(b_{n}\right)\right)=1$.

(c) It holds true

$$
\lim _{\substack{x \rightarrow \infty \\ \lambda \rightarrow 0}} \frac{f(x+\lambda)}{f(x)}=1 .
$$

Proof. Let $f \in \operatorname{Tr}(V D)$. Taking $x(t)=a_{n}$ and $y(t)=b_{n}$ for $n \leq t<$ $n+1$, we have that

$$
\lim _{n \rightarrow \infty} \frac{f\left(a_{n}\right)}{f\left(b_{n}\right)}=\lim _{t \rightarrow \infty} \frac{f\left(a_{[t]}\right)}{f\left(b_{[t]}\right)}=1 .
$$


This means that (a) implies (b). That (b) implies (c), first, take any sequence $x_{n}>0$ tending to $\infty$, and any sequence $\lambda_{n}>0$ tending to 0 as $n \rightarrow \infty$. Second, putting $a_{n}=x_{n}+\lambda_{n}$ and $b_{n}=x_{n}$ we find using (b), that

$$
\lim _{n \rightarrow \infty} \frac{f\left(x_{n}+\lambda_{n}\right)}{f\left(x_{n}\right)}=1
$$

whence we get (c).

Further, by relation (c) we conclude that for every $\varepsilon>0$ there exist some $\gamma(\varepsilon)$ and $\delta(\varepsilon)>0$ such that $|(f(x+\lambda) / f(x))-1|<\varepsilon$ for $x \geq \gamma(\varepsilon)$ and $|\lambda| \leq \delta(\varepsilon)$.

By assumptions from (a), we have that $|x(t)-y(t)| \leq \delta(\varepsilon)$ for $t \geq t_{1}$ and $y(t) \geq \gamma(\varepsilon)$ for $t \geq t_{0}$. Taking $t_{2}=\max \left\{t_{0}, t_{1}\right\}$ we find that $\mid(f(x(t)) / f(y(t))-$ $1 \mid<\varepsilon$ for $t \geq t_{2}$. Thus (c) implies (a). The proof is complete.

Some further facts. In connection with the preceding class $\operatorname{Tr}(V D)$ functions we have an analogous with the Theorems 6 and 9, as a middle solution problem of representation for the classes $\operatorname{Tr}(R V)$ and $\operatorname{Tr}(O R V)$.

Theorem 5.21. A function $f \in \operatorname{Tr}(V D)$ if and only if there exist $\sigma \in$ $\mathbb{R}$ and measurable and bounded real functions $\mu$ and $\eta$ on $I_{b}$ for some $b \geq a$ such that

$$
f(x)=\exp \left(\sigma x+\mu(x)+r(x)+\int_{b}^{x} \eta(t) d t\right)
$$

for $x \geq b$, where $\mu(x) \rightarrow 0$ as $x \rightarrow \infty$ and $r$ is a uniformly continuous bounded function on the interval $I_{b}$ for some $b \geq a$.

Indeed, since the class $\operatorname{Tr}(V D)$ is a proper subclass of the class $\operatorname{Tr}(O R V)$, by Theorem 9 we have that every function $f \in \operatorname{Tr}(O R V)$ has the representation

$$
f(x)=\exp \left(\sigma x+\mu(x)+\int_{b}^{x} \eta(t) d t\right)
$$

for some $\sigma \in \mathbb{R}$ and some measurable bounded functions $\mu$ and $\eta$ on $I_{b}$ for some $b \geq a$. Since $f, g \in \operatorname{Tr}(V D)$ implies that $f \cdot g \in \operatorname{Tr}(V D)$ and since the function.

$$
\psi(x)=\exp \left(\sigma x+\int_{b}^{x} \eta(t) d t\right)
$$

for $x \geq b$ belongs to the class $\operatorname{Tr}(V D)$ we obtain the following fact that is $f \in \operatorname{Tr}(V D)$ if and only if

$$
\lim _{\lambda \rightarrow 0}\left(\lim _{x \rightarrow \infty}(\mu(x+\lambda)-\mu(x))\right)=0,
$$


i.e., from a result of Đurčić [32], the function $\mu(x)$ has a form $h(x)+r(x)$, where $h$ is a measurable bounded function such that $h(x) \rightarrow 0$ as $x \rightarrow \infty$ and $r$ is a bounded and uniformly continuous on $I_{a}$ for some $a>0$.

A general remark. In the preceding parts of this paper we presents main facts on three new classes of functions which have important applications in the study of asymptotic processes and ergodic theory. For further facts of this see: Tasković [29] and [30].

\section{Translational regularly varying sequences}

We notice, in actual fact a weaker definition of (9) can be used, for the assymption that this positive finite limit property obtains for all $\lambda$ in a subset of positive measure of $(0, \infty)$ implies that it obtains for all $\lambda \in(0, \infty)$.

In connection with this, i.e., with $(9)$, since $h(\lambda)$ is a positive measurable solution of the following functional equation

$$
h(u+v)=h(u) h(v), \quad u, v>0,
$$

it is well known that $h(\lambda)=e^{\sigma \lambda}$ for some finite $\sigma$, and so we can write $f(x)=e^{\sigma \lambda} A(x)$, where

$$
\lim _{x \rightarrow \infty} \frac{A(x+\lambda)}{A(x)}=1 \quad \text { for each } \lambda \geq 0 ;
$$

such a translational regularly varying function, for which the index $\sigma$ of translational regular variation is zero, from the former facts, is called translational slowly varying (denoted this class by $\operatorname{Tr}(S V)$ ).

In connection with the preceding facts, a sequence of positive terms $(c(n))$ is said to be translational regularly varying (denoted this class sequences by $\operatorname{Tr}(R V s))$ if

$$
\lim _{n \rightarrow \infty} \frac{c([n+\lambda])}{c(n)}=h(\lambda)
$$

for all $\lambda \geq 0$, where $0<h(\lambda)<\infty$.

It is natural to do this and to expect properties of translational regularly varying sequences so defined to have a theory similar to that for translational regularly varying functions.

It is this last point which is the focus of and motivation for the present paper. We shall show that an analogous theory can be developed from (46), but that this development is not generally close, and sometimes far, from a simple imitation of arguments for translational varying functions, a fact which does not appear to have been hitherto apparent. 
On the other hand, specifically, if $(c(n))$ is a sequence of positive numbers satisfying, for some sequence of positive terms $(\alpha(n))$,

$$
\lim _{n \rightarrow \infty} \frac{c(n)}{\alpha(n)}=\xi \in(0, \infty)
$$

where

$$
\lim _{n \rightarrow \infty} \log \left(\frac{\alpha(n)}{\alpha(n-1)}\right)=\sigma, \quad-\infty<\sigma<\infty,
$$

then $(c(n))$ is said to be a translational regularly varying sequence of index $\sigma$.

If $(c(n))$ is a translational regularly varying sequence in the above sense, the function $f$ defined on $I_{a}$ by $f(x)=c([x])$, is a translational regularly varying function. In this case, make it then possible to apply the results about translational regularly varying functions to the theory of translational regularly varying sequences.

We notice that without this the preceding facts, as we shall see, the theory of translational regularly varying sequences cannot be reconstructed along the lines of the theory of translational regularly varying functions.

According to Karamata definition in [16], a sequence of positive numbers $(c(n))$ is regularly varying if, for some $\rho \in(-1, \infty)$,

$$
\lim _{n \rightarrow \infty} \frac{1}{n c(n)} \sum_{k=1}^{n} c(k)=\frac{1}{\rho+1} .
$$

From this definition one obtains immediately the following representation theorem:

If $(c(n))$ is a sequence of positive numbers such that (47) holds, then there exist sequences $(\eta(n))$ and $(\delta(n))$ coverging to a finite number and zero, respectively, such that

$$
c(n)=n^{\rho} \exp \left(\eta(n)+\sum_{k=1}^{n} \frac{\delta(k)}{k}\right) \quad \text { for } n \in \mathbb{N} .
$$

Former facts. We notice that the elements of a theory of regularly varying sequences were already apparent in the papers of Pólya [24] and Schmidt [25], which to some extent guided Karamata [16], [17] and [18] in his development of the theory of regularly varying sequences and functions. A similar, but slightly more general theory was developed almost at the same time by Schur [28].

A unified theory of regularly varying sequences is given in the paper of Bojanić and Seneta [10]. 
As well as other properties of regularly varying functions and sketch history, were reviewed in Bojanić - Seneta [8].

The following statements of this section are the main results on sequences. In this sense, a first result can be stated as follows.

Theorem 6.22. For a translational regularly varying sequence $(c(n))$ the limit function $h(\lambda)$ has the form $h(\lambda)=e^{\sigma[\lambda]}$ for some finite $\sigma$ and every $\lambda \geq 0$.

In connection with this statement, we can call the exponent $\sigma$ of $h(\lambda)=$ $e^{\sigma[\lambda]}$, arising in connection with a translational regularly varying sequence $(c(n))$, the index of translational regular variation of the sequence. A translational regularly varying sequence of index zero is called a translational slowly varying sequence in denoted this class by $\operatorname{Tr}(S V s)$.

The following immediate consequences of the Theorem 16 are very useful in applications:

Corollary 6.23. A sequence of positive numbers $(c(n))$ is a translational regularly varying sequence of index $\sigma$ if and only if

$$
\lim _{n \rightarrow \infty} \frac{c([n+\lambda])}{c(n)}=e^{\sigma[\lambda]} \quad \text { for every } \lambda \geq 0 .
$$

Corollary 6.24. A sequence of positive numbers $(c(n))$ is a translational regularly varying sequence of index $\sigma$ if and only if

$$
c(n)=e^{\sigma n} A(n), \quad \text { for } n \geq 1,
$$

where $(A(n))$ is a translational slowly varying sequence.

We notice that the proof of Theorem 16 is based on the following two lemmas. In this sense, the proof follows the same ideas as the proof of Theorem 1 in Bojanić-Seneta [10].

Lemma 9. If $(c(n))$ is a translational regularly varying sequence, then we have that $c(n+1) / c(n) \rightarrow 1$ as $n \rightarrow \infty$.

Proof. First write $a(n)=\log (c(n+1) / c(n))$. Then for $\lambda \in(0,1)$, as $n \rightarrow \infty$, we obtain that

$$
\sum_{k=[n+\lambda]}^{n+1} a(k)=\log (c(n+2) / c([n+\lambda])) \rightarrow-\log (h(\lambda) h(2)) ;
$$

and let

$$
A_{n}(\lambda)=\sum_{k=[n+\lambda]}^{n+1} a(k), \quad \text { for } \lambda \in(0,1)
$$


since

$$
A_{n}(\lambda)=\sum_{k=i}^{n+1} a(k) \quad \text { if } \frac{i}{n} \leq \lambda \leq \frac{i+1}{n},
$$

for $i=0,1, \ldots, n-1$, it follows that

$$
A_{n+1}(\lambda)-A_{n}(\lambda)=a(n+2) \quad \text { if } \frac{i}{n} \leq \lambda \leq \frac{i+1}{n+1}
$$

for any $i=0,1, \ldots, n-1$, where it should be noted that

$$
\frac{i}{n+1} \leq \frac{i}{n}<\frac{i+1}{n+1}<\frac{i+1}{n} \text {. }
$$

Define the following set

$$
E_{n}=\bigcup_{i=0}^{n-1}\left(\frac{i}{n}, \frac{i+1}{n+1}\right) \subset(0,1),
$$

so that for any fixed $n$ and $\lambda \in E_{n}$ we have $A_{n+1}(\lambda)-A_{n}(\lambda)=a(n+2)$.

If suppose now that $\xi$ is an irrational number in $(0,1)$, then for given $\varepsilon>0$ we can find $\alpha(\xi, \varepsilon)$ such that for $n \geq \alpha(\xi, \varepsilon)$, we have

$$
\left|A_{n+1}(\xi)-A_{n}(\xi)\right|<\varepsilon \quad \text { and }\left|A_{n+1}(1-\xi)-A_{n}(1-\xi)\right|<\varepsilon \text {. }
$$
then

Hence, for fixed $n \geq \alpha(\xi, \varepsilon)$, there are two possibilities: First, if $\xi \in E_{n}$,

$$
|a(n+2)|=\left|A_{n+1}(\xi)-A_{n}(\xi)\right|<\varepsilon,
$$

and second, if $\xi \notin E_{n}$, then from the definition of $E_{n}$ it follows that

$$
\xi \in \bigcup_{i=1}^{n}\left[\frac{i}{n+1}, \frac{i}{n}\right)
$$

i.e., precise, for some $1 \leq i \leq n$ the following fact holds: $i /(n+1) \leq \xi \leq i / n$, so that

$$
\frac{n-i}{n}<1-\xi \leq \frac{n+1-i}{n+1}
$$

and since $1-\xi$ is irrational, it follows that $1-\xi \in E_{n}$, whence the argument of the first case applies with $1-\xi$ in place of $\xi$. Thus we obtain that

$$
a(n+2)=\log \left(\frac{c(n+3)}{c(n+2)}\right) \rightarrow 0 \quad \text { as }(n \rightarrow \infty)
$$

and thus the statement is proved.

Lemma 10. For a translational regularly varying sequence $(c(n))$ and for fixed $\lambda$ and $\mu$ the following fact holds that is

$$
\lim _{n \rightarrow \infty} \frac{c([n+\mu+\lambda])}{c([n+\mu])}=h(\lambda) .
$$


Proof. Consider first the case where $\lambda \in(0,1)$. For positive integer $n$, $\lambda+\mu+n=\lambda+[n+\mu]+(\lambda+n+\mu)-(\lambda+[n+\mu])$, and since $[x] \leq x<[x]+1$, we obtain

$$
\begin{gathered}
{[\lambda+[n+\mu]] \leq \lambda+[n+\mu] \leq \lambda+\mu+n=} \\
=\lambda+[n+\mu]+(\lambda+n+\mu)-(\lambda+[n+\mu])<[\lambda+[n+\mu]]+1+1 ;
\end{gathered}
$$

hence

$$
[n+\mu+\lambda]=[[n+\mu]+\lambda] \quad \text { or } \quad[n+\mu+\lambda]=[[n+\mu]+\lambda]+1,
$$

and, in the first case we obtain

$$
\frac{c([n+\mu+\lambda])}{c([n+\mu])}=\frac{c([[n+\mu]+\lambda])}{c([n+\mu])}
$$

while in the second case we have

$$
\frac{c([n+\mu+\lambda])}{c([n+\mu])}=\frac{c([[n+\mu]+\lambda]+1)}{c([[n+\mu]+\lambda])} \frac{c([[n+\mu]+\lambda])}{c([n+\mu])} .
$$

In either case the limit of the right-hand side is $h(\lambda)$, in virtue of (46); where in the second case we have used also Lemma 9. If $\lambda \in(1, \infty)$, then we obtain analogous

$$
[[n+\mu]+\lambda] \leq n+\mu+\lambda \leq[[n+\mu]+\lambda]+1+\lambda<[[n+\mu]+\lambda]+2+[\lambda]
$$

so that $[n+\mu+\lambda]$ can take on the values

$$
[[n+\mu]+\lambda],[[n+\mu]+\lambda]+1, \ldots,[[n+\mu]+\lambda]+1+[\lambda]
$$

and repeated use of Lemma 9 finitely many times gives the result in general case. The proof is complete.

Proof of Theorem 16. If we proceed analogously to the function case, then we may write for $0 \leq \lambda, \mu<\infty$ that is

$$
\frac{c([n+\mu+\lambda])}{c(n)}=\frac{c([n+\mu+\lambda])}{c([n+\mu])} \frac{c([n+\mu])}{c(n)} ;
$$

and thus the Theorem 16 can be now proved easily if one observes that the preceding equality, in view of (46) and Lemma 10, implies that $h(\lambda+\mu)=$ $h(\lambda) h(\mu)$, and that $h$ is a positive measurable function. The proof is complete.

We notice that such results, which are also called "imbedding" theorems, make it then possible to apply the results about translational regularly varying functions to the theory of translational regularly varying sequences. The next result is the following extension theorem.

Theorem 6.25. If $(c(n))$ is a translational regularly varying sequence of index $\sigma$ then, the function $f$ defined on $[0, \infty)$ by $f(x)=c([x])$, is a translational regularly varying function of index $\sigma$. 
Proof. Since $(c(n))$ is a translational regularly varying sequence of in$\operatorname{dex} \sigma$, for each $\mu>0$, and fixed $\lambda \geq 0$, by Lemma 10 and Theorem 16 we have

$$
\lim _{n \rightarrow \infty} \frac{c([n+\mu+\lambda])}{c([n+\mu])}=e^{\sigma[\lambda]}
$$

and; since, for fixed $\lambda$,

$$
g_{\lambda}(x)=\frac{c([x+\lambda])}{c([x])}-e^{\sigma[\lambda]}
$$

is a right continuous function in $x>0$, and as $n \rightarrow \infty, g_{\lambda}(n+\mu) \rightarrow 0$ for each $\mu \geq 0$, hence $g_{\lambda}(x) \rightarrow 0$ as $x \rightarrow \infty$ through all real values. Thus for each $\lambda \geq 0$ we obtain that is

$$
\lim _{x \rightarrow \infty} \frac{c([x+\lambda])}{c([x])}=e^{\sigma[\lambda]},
$$

so $f(x)=c([x])$ is clearly a translational regularly varying function of index $\sigma$. The proof is complete.

In connection with the preceding statement, since we may now apply for $f$ all the properties possessed by translational regularly varying functions, it is easy to deduce some of the analogous properties for the sequence $(c(n))$. In this sense we have the following essential statement.

Theorem 6.26. (Representation Theorem). If $(c(n))$ is a translational regularly varying sequence of index $\sigma$, then there exist sequences $(\mu(n))$ and $(\delta(n))$ coverging to a finite limit and zero, respectively, such that

$$
c(n)=\mu(n) \exp \left(\sigma n+\sum_{k=1}^{n} \delta(k)\right)
$$

for $n \in \mathbb{N}$. Conversely, such a representation for a sequence $(c(n))$ implies it is translational regularly varying of index $\sigma$.

In connection with the preceding facts, from Theorem 18, as an immediate consequence, in particular, we have that

$$
e^{-\rho n} c(n) \rightarrow \infty \quad \text { for every } \rho<\sigma
$$

and

$$
e^{-\tau n} c(n) \rightarrow 0 \text { for every } \tau>\sigma .
$$

Proof of Theorem 18. This proof follows from Theorem 17 and the Representation Theorem for translational regularly varying functions.

If $(c(n))$ is a translational regularly varying sequence of index $\sigma$, by Theorem 17 , the function $f(x)=c([x])$ is a translational regularly varying 
function of index $\sigma$. It follows then that there exists a positive integer $b \geq a$ such that for $x \geq b$ we have

$$
f(x)=\mu(x) \exp \left(\sigma x+\int_{b}^{x} \varepsilon(t) d t\right),
$$

where $\mu(x)$ is a positive and measurable function on $I_{b}$ such that $\mu(x) \rightarrow c \in$ $(0, \infty)$ as $x \rightarrow \infty$ and $\varepsilon(x)$ is a continuous function on $I_{b}$ such that $\varepsilon(x) \rightarrow 0$ as $x \rightarrow \infty$. Thus, for $n \geq b$ we have

or

$$
c(n)=f(n)=\mu(n) \exp \left(\sigma n+\int_{b}^{n} \varepsilon(t) d t\right)
$$

$$
c(n)=\mu(n) \exp \left(\sigma n+\sum_{k=b+1}^{n} \delta(k)\right)
$$

where

$$
\delta(k)=\int_{k-1}^{k} \varepsilon(t) d t, \quad \text { for } k \geq b+1 ;
$$

and, we have, as $n \rightarrow \infty, \mu(n) \rightarrow c \in(0, \infty)$ and

$$
|\delta(n)| \leq\left(\sup _{t \geq n-1}|\varepsilon(t)|\right) \int_{n-1}^{n} d t=\sup _{t \geq n-1}|\varepsilon(t)| \rightarrow 0 .
$$

Hence, finally, an obvious extension of sequences $(\mu(n))$ and $(\delta(n))$ for $1 \leq n \leq b$ completes the first part of the proof of Theorem 18 .

On the other hand, conversely, if a sequence $(c(n))$ has the representation (48), then we have, for each $\lambda \geq 0$, as $n \rightarrow \infty$,

$$
\frac{c([n+\lambda])}{c(n)}=\frac{\mu([n+\lambda])}{\mu(n)} \exp \left(\sigma\{[n+\lambda]-n\}+\sum_{k=n}^{[n+\lambda]} \delta(k)\right) \rightarrow e^{\sigma[\lambda]},
$$

since $[n+\lambda]-n \rightarrow[\lambda]$ and

$$
\sum_{k=n}^{[n+\lambda]} \delta(k) \leq\left(\sup _{k \geq n}|\delta(k)|\right)\{[n+\lambda]-n\} \rightarrow 0 .
$$

Theorem 6.27. A sequence $(c(n))$ of positive numbers is a translational regularly varying sequence of index $\sigma$ if and only if there is a sequence of positive numbers $(\alpha(n))$ such that

$$
\lim _{n \rightarrow \infty} \frac{c(n)}{\alpha(n)}=\xi \in(0, \infty)
$$

where

$$
\log \left(\frac{\alpha(n)}{\alpha(n-1)}\right) \rightarrow \sigma \quad \text { as } n \rightarrow \infty
$$


Proof. We shall show first that conditions (49) and (50) of statement imply the representation (48) for $(c(n))$, so the sufficiency part of Theorem 19 follows from Theorem 18. Let

$$
\rho(n)=\log \left(\frac{\alpha(n)}{\alpha(n-1)}\right) \quad \text { for } n \in \mathbb{N},
$$

where $\alpha(0)=1$. By (50) we have $\rho(n) \rightarrow \sigma(n \rightarrow \infty)$. From (51) follows that, for $n \geq 1$, we obtain

$$
\frac{\alpha(n)}{\alpha(n-1)}=\exp \rho(n)
$$

i.e.,

$$
\begin{aligned}
\log \alpha(n) & =\rho(1)+\cdots+\rho(n)=\sum_{k=1}^{n} \rho(k)= \\
& =\sum_{k=1}^{n}(\rho(k)-\sigma)+\sigma n
\end{aligned}
$$

and, finally,

$$
\begin{aligned}
c(n) & =\exp \left(\log \frac{c(n)}{\alpha(n)}+\log \alpha(n)\right)= \\
& =\mu(n) \exp \left(\sigma n+\sum_{k=1}^{n} \delta(k)\right),
\end{aligned}
$$

where $\mu(n)=c(n) / \alpha(n)$ is a convergent sequence and $\delta(n)=\rho(n)-\sigma \rightarrow 0$ $(n \rightarrow \infty)$.

Converselly, if $(c(n))$ is a translational regularly varying sequence of index $\sigma$, then, from Theorem 18, we have (48). If $\mu(n) \rightarrow \xi \in(0, \infty)$ as $n \rightarrow \infty$, let $\alpha(n)$ by defined by

$$
\alpha(n)=\exp \left(\sigma n+\sum_{k=1}^{n} \delta(k)\right) \quad \text { for } n \in \mathbb{N} ;
$$

then (49) clearly holds and

$$
\log \left(\frac{\alpha(n)}{\alpha(n-1)}\right)=\log (\exp \{\sigma+\delta(n)\})=\sigma+\delta(n) \rightarrow \sigma
$$

as $n \rightarrow \infty$, i.e., (50) follows. The proof is complete.

Theorem 6.28. A sequence of positive numbers $(c(n))$ is translational regularly varying of index $\sigma$ if and only if, as $n \rightarrow \infty$, 
(i) ${ }^{7)}$ for every $\rho<\sigma$ :

$$
\max _{1 \leq k \leq n}\left\{e^{-\rho k} c(k)\right\} \sim e^{-\rho n} c(n)
$$

and

$$
\inf _{k \geq n}\left\{e^{-\rho k} c(k)\right\} \sim e^{-\rho n} c(n)
$$

and

(ii) for every $\tau>\sigma$ :

$$
\min _{1 \leq k \leq n}\left\{e^{-\tau k} c(k)\right\} \sim e^{-\tau n} c(n)
$$

and

$$
\sup _{k \geq n}\left\{e^{-\tau k} c(k)\right\} \sim e^{-\tau n} c(n) .
$$

Proof. First suppose that $(c(n))$ is a translational regularly varying sequence of index $\sigma$. We have that is

$$
\limsup _{n \rightarrow \infty}\left(\frac{e^{-\rho n} c(n)}{\max _{1 \leq k \leq n}\left\{e^{-\rho k} c(k)\right\}}\right) \leq 1,
$$

and, thus, in order to prove the first of the relations (i), it will be sufficient to show that

$$
\liminf _{n \rightarrow \infty}\left(\frac{e^{-\rho n} c(n)}{\max _{1 \leq k \leq n}\left\{e^{-\rho k} c(k)\right\}}\right) \geq 1 .
$$

If this inequality were not true, there would exist sequences $\left(a_{i}\right)$ and $\left(b_{i}\right)$ of positive integers such that $1 \leq b_{i} \leq a_{i}$, where $b_{i} \rightarrow \infty$ as $i \rightarrow \infty$ in view of fact that $e^{-\rho n} c(n) \rightarrow \infty$ for every $\rho<\sigma$ and

$$
\lim _{i \rightarrow \infty} \frac{e^{-\rho a_{i}} c\left(a_{i}\right)}{e^{-\rho b_{i}} c\left(b_{i}\right)}=d<1 .
$$

Since $e^{-\rho n} c(n)=\exp (\{\sigma-\rho\} n) A(n)$ is a translational regularly varying sequence of index $\sigma-\rho>0$, by Theorem 19 we can find a sequence of positive numbers $(\alpha(n))$ such that

$$
\frac{e^{-\rho n} c(n)}{\alpha(n)} \rightarrow \xi \in(0, \infty) \quad \text { and } \quad \log \left(\frac{\alpha(n)}{\alpha(n-1)}\right) \rightarrow \sigma-\rho
$$

as $n \rightarrow \infty$. In view of the preceding relations, the sequence $(\alpha(n))$ is eventually nondecreasing. Writing $e^{-\rho n} c(n)=a(n) \alpha(n)$, we have

$$
\lim _{i \rightarrow \infty} \frac{\alpha\left(a_{i}\right)}{\alpha\left(b_{i}\right)}=\lim _{i \rightarrow \infty} \frac{a\left(b_{i}\right)}{a\left(a_{i}\right)} \cdot \frac{e^{-\rho a_{i}} c\left(a_{i}\right)}{e^{-\rho b_{i}} c\left(b_{i}\right)}=d<1,
$$

\footnotetext{
${ }^{7)} a_{n} \sim b_{n}(n \rightarrow \infty)$ means $a_{n} / b_{n} \rightarrow 1(n \rightarrow \infty)$.
} 
which is impossible since $\alpha\left(a_{i}\right) \geq \alpha\left(b_{i}\right)$. To prove the second of the relations (i), observe first that

$$
\liminf _{n \rightarrow \infty}\left(\frac{e^{-\rho n} c(n)}{\inf _{k \geq n}\left\{e^{-\rho k} c(k)\right\}}\right) \geq 1 .
$$

and suppose that

$$
\limsup _{n \rightarrow \infty}\left(\frac{e^{-\rho n} c(n)}{\inf _{k \geq n}\left\{e^{-\rho k} c(k)\right\}}\right)=d>1 ;
$$

and, since $e^{-\rho n} c(n) \rightarrow \infty(n \rightarrow \infty)$ by Theorem 18, it is easy to see that $\inf _{k \geq n}\left\{e^{-\rho k} c(k)\right\}$ is actually attained for some $k \geq n$ and for each $n \in \mathbb{N}$. From here, the proof is analogous to the preceding proof. The proof of relations (ii) of statement is similar and hence omitted.

Next, suppose that $(c(n))$ is a sequence of positive numbers satisfying (i) and (ii) of statement. We have

$$
\begin{gathered}
\frac{c([n+\lambda])}{c(n)} \geq \frac{c([n+\lambda])}{e^{\rho n} \max _{1 \leq k \leq[n+\lambda]}\left\{e^{-\rho k} c(k)\right\}}= \\
\quad=\frac{e^{\rho[n+\lambda]}}{e^{\rho n}}\left(\frac{e^{-\rho[n+\lambda]} c([n+\lambda])}{\max _{1 \leq k \leq[n+\lambda]}\left\{e^{-\rho k} c(k)\right\}}\right)
\end{gathered}
$$

and, hence, by the first of the realtions (i) of statement we obtain

$$
\liminf _{n \rightarrow \infty} \frac{c([n+\lambda])}{c(n)} \geq e^{\rho[\lambda]}
$$

and, on the other hand, next

$$
\begin{gathered}
\frac{c([n+\lambda])}{c(n)} \leq \frac{c([n+\lambda])}{e^{\tau n} \min _{1 \leq k \leq[n+\lambda]}\left\{e^{-\tau k} c(k)\right\}}= \\
=\frac{e^{\tau[n+\lambda]}}{e^{\tau n}}\left(\frac{e^{-\tau[n+\lambda]} c([n+\lambda])}{\min _{1 \leq k \leq[n+\lambda]}\left\{e^{-\tau k} c(k)\right\}}\right)
\end{gathered}
$$

and so, by the first of the realtions (ii) of statement, we obtain

$$
\limsup _{n \rightarrow \infty} \frac{c([n+\lambda])}{c(n)} \leq e^{\tau[\lambda]},
$$

i.e., since $\rho<\sigma<\tau$ can be chosen arbitrarily close to $\sigma$ it follows that

$$
\lim _{n \rightarrow \infty} \frac{c([n+\lambda])}{c(n)}=e^{\sigma[\lambda]}
$$

for every $\lambda \geq 0$. Now the proof is complete. (For this see and: Tasković [29].) 
Some applications. From the preceding statements we are now in a position to formulate the following consequences:

(a) If $(c(n))$ is a translational regularly varying sequence of index $\sigma$, then the following inequalities hold that is

$$
\limsup _{n \rightarrow \infty} \frac{1}{e^{-\rho n} c(n)} \sum_{k=1}^{n} e^{\alpha k} c(k) \leq \frac{e^{\alpha+\rho}}{1-e^{\alpha+\rho}}
$$

for some $\alpha+\rho<0$ and $\rho<\sigma$; and that is

$$
\liminf _{n \rightarrow \infty} \frac{1}{e^{-\tau n} c(n)} \sum_{k=1}^{n} e^{\alpha k} c(k) \geq \frac{e^{\alpha+\tau}}{1-e^{\alpha+\tau}}
$$

for some $\alpha+\tau<0$ and $\sigma<\tau$.

(b) If $(c(n))$ is a translational regularly varying sequence of index $\sigma$, then the following inequalities hold that is:

$$
\limsup _{n \rightarrow \infty} \frac{1}{e^{\alpha n} c(n)} \sum_{k=n}^{\infty} e^{\alpha k} c(k) \leq \frac{1}{1-e^{\alpha+\tau}}
$$

for some $\alpha+\tau<0$ and $\sigma<\tau$; and that is

$$
\liminf _{n \rightarrow \infty} \frac{1}{e^{\alpha n} c(n)} \sum_{k=n}^{\infty} e^{\alpha k} c(k) \geq \frac{1}{1-e^{\alpha+\rho}}
$$

for some $\alpha+\rho<0$ and $\rho<\sigma$.

Proofs. Assume that $(c(n))$ is a translational regularly varying sequence with index $\sigma$. Let $\alpha+\rho<0$ and let $\rho<\sigma$. We have then

$$
\begin{aligned}
& \sum_{k=1}^{n} e^{\alpha k} c(k)=\sum_{k=1}^{n} e^{(\alpha+\rho) k} e^{-\rho k} c(k) \leq \\
& \leq\left\{\max _{1 \leq k \leq n}\left(e^{-\rho k} c(k)\right)\right\} \sum_{k=1}^{n} e^{(\alpha+\rho) k} ;
\end{aligned}
$$

and since $\alpha+\rho<0$ and $\rho<\sigma$, we have

$$
\sum_{k=1}^{n} e^{(\alpha+\rho) k}=e^{\alpha+\rho} \frac{1-e^{(\alpha+\rho) n}}{1-e^{\alpha+\rho}}
$$

hence

$$
\frac{1}{e^{-\rho n} c(n)} \sum_{k=1}^{n} e^{\alpha k} c(k) \leq\left(\frac{\max _{1 \leq k \leq n}\left(e^{-\rho k} c(k)\right)}{e^{-\rho n} c(n)}\right) e^{\alpha+\rho} \frac{1-e^{(\alpha+\rho) n}}{1-e^{\alpha+\rho}},
$$

and so, by the first of the relations (i) of Theorem 20, we obtain

$$
\limsup _{n \rightarrow \infty} \frac{1}{e^{-\rho n} c(n)} \sum_{k=1}^{n} e^{\alpha k} c(k) \leq \frac{e^{\alpha+\rho}}{1-e^{\alpha+\rho}}
$$


Likewise, if $\alpha+\tau<0$ and $\tau>\sigma$, then we have

$$
\begin{aligned}
& \sum_{k=1}^{n} e^{\alpha k} c(k)=\sum_{k=1}^{n} e^{(\alpha+\tau) k} e^{-\tau k} c(k) \geq \\
& \geq\left\{\min _{1 \leq k \leq n}\left(e^{-\tau k} c(k)\right)\right\} \sum_{k=1}^{n} e^{(\alpha+\tau) k} ;
\end{aligned}
$$

and since

$$
\sum_{k=1}^{n} e^{(\alpha+\tau) k}=e^{\alpha+\tau} \frac{1-e^{(\alpha+\tau) n}}{1-e^{\alpha+\tau}}
$$

hence

$$
\frac{1}{e^{-\tau n} c(n)} \sum_{k=1}^{n} e^{\alpha k} c(k) \geq\left(\frac{\min _{1 \leq k \leq n}\left(e^{-\tau k} c(k)\right)}{e^{-\tau n} c(n)}\right) e^{\alpha+\tau} \frac{1-e^{(\alpha+\tau) n}}{1-e^{\alpha+\tau}}
$$

and, so by the first of the relations (ii) of Theorem 20 we obtain

$$
\liminf _{n \rightarrow \infty} \frac{1}{e^{-\tau n} c(n)} \sum_{k=1}^{n} e^{\alpha k} c(k) \geq \frac{e^{\alpha+\tau}}{1-e^{\alpha+\tau}},
$$

i.e., (a) holds. On the other hand, if $(c(n))$ is a translational regularly varying sequence with index $\sigma$ and if $\alpha+\tau<0$ and $\sigma<\tau$, then we have

$$
\begin{gathered}
\sum_{k=n}^{\infty} e^{\alpha k} c(k)=\sum_{k=n}^{\infty} e^{(\alpha+\tau) k} e^{-\tau k} c(k) \leq \\
\leq\left(\sup _{k \geq n}\left\{e^{-\tau k} c(k)\right\}\right) \sum_{k=n}^{\infty} e^{(\alpha+\tau) k} ;
\end{gathered}
$$

and since $\alpha+\tau<0$ and $\tau>\sigma$, we have

$$
\sum_{k=n}^{\infty} e^{(\alpha+\tau) k}=e^{(\alpha+\tau) n} \sum_{k=0}^{\infty} e^{(\alpha+\tau) k}
$$

hence

$$
\frac{1}{e^{\alpha n} c(n)} \sum_{k=n}^{\infty} e^{\alpha k} c(k) \leq\left(\frac{\sup _{k \geq n}\left(e^{-\tau k} c(k)\right)}{e^{-\tau n} c(n)}\right) \sum_{k=0}^{\infty} e^{(\alpha+\tau) k}
$$

and so, by the second of the relations (ii) of Theorem 20, we obtain

$$
\limsup _{n \rightarrow \infty} \frac{1}{e^{\alpha n} c(n)} \sum_{k=n}^{\infty} e^{\alpha k} c(k) \leq \frac{1}{1-e^{\alpha+\tau}} .
$$


Likewise, if $\alpha+\rho<0$ and $\rho<\sigma$, then we have that is

$$
\begin{gathered}
\sum_{k=n}^{\infty} e^{\alpha k} c(k)=\sum_{k=n}^{\infty} e^{(\alpha+\rho) k} e^{-\rho k} c(k) \geq \\
\geq\left\{\inf _{k \geq n}\left(e^{-\rho k} c(k)\right)\right\} \sum_{k=n}^{\infty} e^{(\alpha+\rho) k} ;
\end{gathered}
$$

and since

$$
\sum_{k=n}^{\infty} e^{(\alpha+\rho) k}=e^{(\alpha+\rho) n} \sum_{k=0}^{\infty} e^{(\alpha+\rho) k}
$$

hence

$$
\frac{1}{e^{\alpha n} c(n)} \sum_{k=n}^{\infty} e^{\alpha k} c(k) \geq\left(\frac{\inf _{k \geq n}\left\{e^{-\rho k} c(k)\right\}}{e^{-\rho n} c(n)}\right) \sum_{k=0}^{\infty} e^{(\alpha+\rho) k}
$$

and so, by the second of the relations (i) of Theorem 20, we obtain

$$
\liminf _{n \rightarrow \infty} \frac{1}{e^{\alpha n} c(n)} \sum_{k=n}^{\infty} e^{\alpha k} c(k) \geq \frac{1}{1-e^{\alpha+\rho}} ;
$$

which means that the proof, of (a) and (b) of the preceding statements, is complete.

\section{Translational $\mathcal{O}$-regularly varying sequences}

In connection with the preceding facts, a sequence of positive terms $(c(n))$ is said to be translational $\mathcal{O}$-regularly varying (denoted this class by $\operatorname{Tr}(O R V s))$ if

$$
\limsup _{n \rightarrow \infty} \frac{c([n+\lambda])}{c(n)}=r(\lambda)
$$

for all $\lambda \geq 0$, where $0<r(\lambda)<\infty$.

It is natural to do this and to expect properties of translational $\mathcal{O}$ regularly varying sequences so defined to have a theory similar to that for translational $\mathcal{O}$-regularly varying functions.

If $(c(n))$ is a translational $\mathcal{O}$-regularly varying sequence in the above sense, the function $f$ defined on $I_{a}$ by $f(x)=c([x])$, is a translational $\mathcal{O}$ regularly varying function. Make it then possible to apply the results about translational $\mathcal{O}$-regularly varying functions to the theory of translational $\mathcal{O}$ regularly varying sequences. In this sense we have the following extension theorem. 
Theorem 7.29. If $(c(n))$ is a translational $\mathcal{O}$-regularly varying sequence then the function $f$, defined on $[0,+\infty)$ by $f(x)=c([x])$, is a translational $\mathcal{O}$-regularly varying function.

Proof for this statement is analogous to the proof of Theorem 17.

In connection with this statement, since we may now apply for $f$ all the properties possessed by translational $\mathcal{O}$-regularly varying functions, it is easy to deduce some of the analogous properties for the sequence $(c(n))$. Thus we have the following essential fact.

Theorem 7.30. (Representation Theorem). If $(c(n))$ is a translational $\mathcal{O}$-regularly varying sequence, then there exist $\sigma \in \mathbb{R}$ and bounded sequences $(\mu(n))$ and $(\delta(n))$ such that

$$
c(n)=\exp \left(\sigma n+\mu(n)+\sum_{k=1}^{n} \delta(k)\right)
$$

for $n \in \mathbb{N}$. Conversely, such a representation for a sequence $(c(n))$ implies it is translational $\mathcal{O}$-regularly varying.

The proof of this statement follows from Theorem 21 and the Representation Theorem for translational $\mathcal{O}$-regularly varying functions.

\section{Some subclasses of the class $\operatorname{Tr}(O R V s)$}

From the preceding facts we have the following relations: $\operatorname{Tr}(S V s) \subset$ $\operatorname{Tr}(R V s) \subset \operatorname{Tr}(O R V s)$. In this sense we give a number new examples of subclasses sequences of the class $\operatorname{Tr}(O R V s)$.

Example 4. $(\operatorname{Tr}(E R V s)$ sequences $)$. A sequence of positive terms $(c(n))$ is said to be translational extended regular variation (denoted this class by $\operatorname{Tr}(E R V s))$ if

$$
\exp (\lambda d) \leq \liminf _{n \rightarrow \infty} \frac{c([n+\lambda])}{c(n)} \leq \limsup _{n \rightarrow \infty} \frac{c([n+\lambda])}{c(n)} \leq \exp (\lambda \rho)
$$

for some $d, \rho \in \mathbb{R}$ and for every $\lambda \geq 0$. We have clearly $\operatorname{Tr}(R V s) \subset \operatorname{Tr}(E R V s) \subset$ $\operatorname{Tr}(O R V s)$, i.e., $\operatorname{Tr}(E R V s)$ is a subclass of the class of all $\operatorname{Tr}(O R V s)$ sequences.

Remark. We notice, an intermediary class of sequences of extended regular variation (denoted by ERVs) was found by Matuszewska-Orlicz [23]. In this sense, a sequence of positive terms $(c(n))$ is said to be $E R V s$ if

$$
\lambda^{d} \leq \liminf _{n \rightarrow \infty} \frac{c([\lambda n])}{c(n)} \leq \limsup _{n \rightarrow \infty} \frac{c([\lambda n])}{c(n)} \leq \lambda^{\rho}
$$


for some $d, \rho \in \mathbb{R}$ and for every $\lambda \geq 1$. As well-known $S V s \subset R V s \subset E R V s \subset$ ORVs.

Example 5. $\left(\operatorname{Tr}\left(S_{c *}\right)\right.$ sequences $)$. A sequence of positive terms $(c(n))$ is said to be translational $S_{c *}$ varying (denoted this class by $\operatorname{Tr}\left(S_{c *}\right)$ ) if

$$
\lim _{n \rightarrow \infty} \frac{c([n+\psi(n)])}{c(n)}=1
$$

for every continuous function $\psi: I_{a} \rightarrow(0, \infty)$ tending to zero as $x \rightarrow \infty$. Thus we have $\operatorname{Tr}\left(S_{c *}\right) \subset \operatorname{Tr}(O R V s)$.

On the other hand, in special case, a sequence of positive terms $(c(n))$ is said to be translational $*$-regularly varying (denoted this class by $\operatorname{Tr}(* R V s)$ ) if

$$
\lim _{\lambda \rightarrow 0}\left(\lim _{n \rightarrow \infty} \frac{c([n+\lambda])}{c(n)}\right)=1 ;
$$

and, also, in this sense a sequence of positive terms $(c(n))$ is said to be translational $* \mathcal{O}$-regularly varying (denoted this class by $\operatorname{Tr}(* O R V s)$ ) if

$$
\lim _{\lambda \rightarrow 0}\left(\limsup _{n \rightarrow \infty} \frac{c([n+\lambda])}{c(n)}\right)=1 .
$$

From the preceding facts we have the following essential statement which is connected with the former Theorem 15.

Theorem 8.31. (Representation Theorem). If $(c(n))$ is a translational $* \mathcal{O}$-regularly varying sequence, then there exist $\sigma \in \mathbb{R}$ and bounded sequences $(\mu(n)),(r(n))$ and $(\delta(n))$ such that

$$
c(n)=\exp \left(\sigma n+\mu(n)+r(n)+\sum_{k=1}^{n} \delta(k)\right)
$$

for $n \in \mathbb{N}$, where $r$ is a uniformly continuous function on the interval $I_{s}$ for some $s \in \mathbb{N}$. Conversely, such a representation for a sequence $(c(n))$ implies it is translational $* \mathcal{O}$-regularly varying.

The proof (in main line) of this statement is analogous to the proof of Theorems 15 and 18.

\section{Some open problems and an illustration}

We shall say that a positive, finite and measurable function $f$, defined on $I_{a}$ for some $a>0$, is a translational homothetic function at infinity (denoted this class by $T H$ ) if the limit

$$
\lim _{x \rightarrow \infty} \frac{f(\lambda x+\tau)}{f(x)}=k(\lambda, \tau)
$$


is positive and finite for all $\lambda>0$ and $\tau \geq 0$. Thus, from (58), for $\lambda=1$ we obtain the class $\operatorname{Tr}(R V)$ and for $\tau=0$ we have the class $R V$ in Karamata's sense.

Open problem 1. To invent representation of the class TH of the all translational homothetic functions!? Also, to invent and some characterizations of the class functions TH!?

We notice, from (58) we obtain the following functional equation in the form

$$
k(\lambda, \tau)=k(1, \tau) k(\lambda, 0)
$$

for all $\lambda>0$ and all $\tau \geq 0$. This is an essential fact for further solution of open problem 1 .

In connection with the preceding, parallel to the $T H$ functions we can consider $O T H$ functions.

In this sense, a positive, finite and measurable function $f$ on $I_{a}$ for some $a>0$ is said to be $\mathcal{O}$-translational homothetic at infinity (denoted this class by $O T H$ ) if

$$
\limsup _{x \rightarrow \infty} \frac{f(\lambda x+\tau)}{f(x)}=r(\lambda, \tau)
$$

is finite for all $\lambda>0$ and $\tau \geq 0$. Specially, from (59), for $\lambda=1$ we have defined the class $\operatorname{Tr}(O R V)$ and for $\tau=0$ we, from the preceding equality, obtain defined the class $O R V$ in the Karamata's and Avakumović's sense.

Open problem 2. To invent representation of the class OTH of the all $\mathcal{O}$-translational homothetic functions!? In this sense, to invent and some characterizations of the class functions OTH!?

In connection with this, we notice that, from

$$
\frac{f(\lambda x+\tau)}{f(x)}=\frac{f(\lambda x+\tau)}{f(\lambda x)} \frac{f(\lambda x)}{f(x)}
$$

there follows, as $x \rightarrow \infty$,

$$
r(\lambda, \tau) \leq r(1, \tau) r(\lambda, 0)
$$

for all $\lambda>0$ and all $\tau \geq 0$. This is an essential fact for further solution of open problem 2 .

As a speciall class functions we defined the following subclass of the class $O T H$ functions. In this sense, a positive finite and measurable function $f \mid I_{a}$ for some $a>0$ is said to be translational $S_{c}$ homothetic at infinity (denoted this class by $\left.T H S_{c}\right)$ if the limit

$$
\lim _{x \rightarrow \infty} \frac{f(\mu(x) x+\psi(x))}{f(x)}=1
$$


for all continuos functions $\mu, \psi: I_{a} \rightarrow(0, \infty)$ where $\mu(x) \rightarrow 1$ and $\psi(x) \rightarrow 0$ as $x \rightarrow \infty$.

Thus, from (60), for $\mu(x) \equiv 1$ we obtain the class $\operatorname{Tr}\left(S_{c}\right)$ functions (see example 2). If $\psi(x) \equiv 0$, then we have the class $K_{c}$ functions from Schmidt [25] and Matuszewska - Orlicz [23].

Parallel to the $T H S_{c}$ functions we can consider $O T H *$ functions and $O H T *$ functions. Namely, the class $T H *$ functions (or HT* functions) it is the class of all functions $f \mid I_{a}$ for some $a>0$ which satisfying the following equality

$$
\lim _{\substack{\tau \rightarrow 0 \\ \lambda \rightarrow 1}}\left(\lim _{x \rightarrow \infty} \frac{f(\lambda x+\tau)}{f(x)}\right)=1 \quad \text { or } \quad \lim _{\substack{\lambda \rightarrow 1 \\ \tau \rightarrow 0}}\left(\lim _{x \rightarrow \infty} \frac{f(\lambda x+\tau)}{f(x)}\right)=1 ;
$$

and the class $O T H *$ functions (or OHT* functions) it is the class of all functions $f \mid I_{a}$ for some $a>0$ which satisfying the following equality

$$
\lim _{\substack{\tau \rightarrow 0 \\ \lambda \rightarrow 1}}\left(\limsup _{x \rightarrow \infty} \frac{f(\lambda x+\tau)}{f(x)}\right)=1 \quad \text { or } \quad \lim _{\substack{\lambda \rightarrow 1 \\ \tau \rightarrow 0}}\left(\limsup _{x \rightarrow \infty} \frac{f(\lambda x+\tau)}{f(x)}\right)=1 .
$$

Open problem 3. To invent representation of the class TH* functions (or HT* functions)! Also, to invent represantation of the class OTH* functions (or OHT* functions)!?

In the context with the preceding facts of this paper the following classes of functions are of interest for further considered. Namely, it are the classes of all functions $f \mid I_{a}$ for some $a>0$ which satisfying the following equalities

$$
\lim _{\lambda \rightarrow 1}\left(\lim _{x \rightarrow \infty} \frac{f(\lambda x+\tau)}{f(x)}\right)=1 \quad \text { or } \quad \lim _{\lambda \rightarrow 1}\left(\limsup _{x \rightarrow \infty} \frac{f(\lambda x+\tau)}{f(x)}\right)=1
$$

for every $\tau \geq 0$, and, on the other hand, which satisfying the following equalities

$$
\lim _{\tau \rightarrow 0}\left(\lim _{x \rightarrow \infty} \frac{f(\lambda x+\tau)}{f(x)}\right)=1 \quad \text { or } \quad \lim _{\tau \rightarrow 0}\left(\limsup _{x \rightarrow \infty} \frac{f(\lambda x+\tau)}{f(x)}\right)=1
$$

for every $\lambda>0$. Does some of this classes functions equally of the some of the classes functions introduced in this paper!?

In connection with this, the following classes of sequences are of interest for further considered, i.e., it are the classes of all sequences of positive terms $(c(n))$ which satisfying

$$
\lim _{\lambda \rightarrow 1}\left(\lim _{n \rightarrow \infty} \frac{c([\lambda n+\tau])}{c(n)}\right)=1 \quad \text { or } \quad \lim _{\lambda \rightarrow 1}\left(\limsup _{n \rightarrow \infty} \frac{c([\lambda n+\tau])}{c(n)}\right)=1
$$

for every $\tau \geq 0$, and, on the other hand, which satisfying the following equalities

$$
\lim _{\tau \rightarrow 0}\left(\lim _{n \rightarrow \infty} \frac{c([\lambda n+\tau])}{c(n)}\right)=1 \quad \text { or } \quad \lim _{\tau \rightarrow 0}\left(\limsup _{n \rightarrow \infty} \frac{c([\lambda n+\tau])}{c(n)}\right)=1
$$


for very $\lambda>0$. Does some of this classes sequences equally of the some of the classes sequences introduced in this paper?!

In connection with the preceding facts, a sequence of positive terms $(c(n))$ is said to be translational homothetic (denoted this class by $T H s$ ) if

$$
\lim _{n \rightarrow \infty} \frac{c([\lambda n+\tau])}{c(n)}=h(\lambda, \tau)
$$

for all $\lambda>0$ and $\tau \geq 0$, where $0<h(\lambda, \tau)<\infty$. On the other hand, a sequence of positive terms $(c(n))$ is said to be $\mathcal{O}$-translational homothetic (denoted this class by $O T H s)$ if

$$
\limsup _{n \rightarrow \infty} \frac{c([\lambda n+\tau])}{c(n)}=r(\lambda, \tau)
$$

for all $\lambda>0$ and $\tau \geq 0$, where $0<r(\lambda, \tau)<\infty$.

It is natural to do this and to expect properties of OTHs (or THs) sequences so defined to have a theory similar to that for $O T H$ (or $T H$ ) functions.

Open problem 4. To invent representation of the class THs of the all translational homothetic sequences!? Also, to invent representation of the class OTHs of the all $\mathcal{O}$-translational homothetic sequences!?

Example 6. (TH $S_{c *}$ sequences). A sequence of positive terms $(c(n))$ is said to be translational $S_{c *}$ homothetic (denoted this class by $T H S_{c *}$ ) if

$$
\lim _{n \rightarrow \infty} \frac{c([\mu(n) n+\psi(n)])}{c(n)}=1
$$

for all continuous functions $\mu, \psi: I_{a} \rightarrow(0, \infty)$, where $\mu(x) \rightarrow 1$ and $\psi(x) \rightarrow 0$ as $x \rightarrow \infty$.

On the other hand, in special cases, a sequence of positive terms $(c(n))$ is said to be $*$-translational homothetic (or *-homothetic translational) denoted this class by $* T H s($ or $* H T s)$ if

$$
\lim _{\substack{\tau \rightarrow 0 \\ \lambda \rightarrow 1}}\left(\lim _{n \rightarrow \infty} \frac{c([\lambda n+\tau])}{c(n)}\right)=1 \quad \text { or } \quad \lim _{\substack{\lambda \rightarrow 1 \\ \tau \rightarrow 0}}\left(\lim _{n \rightarrow \infty} \frac{c([\lambda n+\tau])}{c(n)}\right)=1 ;
$$

and, also, a sequence of positive terms $(c(n))$ is said to be $* \mathcal{O}$-translational homothetic (or $* \mathcal{O}$-homothetic translational) denoted this class by $* O T H s$ (or $* O H T s)$ if

$$
\lim _{\substack{\tau \rightarrow 0 \\ \lambda \rightarrow 1}}\left(\limsup _{n \rightarrow \infty} \frac{c([\lambda n+\tau])}{c(n)}\right)=1 \quad \text { or } \quad \lim _{\substack{\lambda \rightarrow 1 \\ \tau \rightarrow 0}}\left(\limsup _{n \rightarrow \infty} \frac{c([\lambda n+\tau])}{c(n)}\right)=1 .
$$

Open problem 5. To invent representation of the class $T H S_{c *}$ sequences!? Also, to invent representations of the classes. $* T H s, * H T s, * O T H s$ and $* O H T s$ sequences!? 
An illustration and application. We notice that Tauberian statements are concerned with the deduction of the asymptotic behaviour of functions from the asymptotic behaviour of their transforms. In this sense, a typical result of the Tauberian nature can be stated as follows.

Let $G(x)$, defined and positive on $I_{a}$ for some $a>0$, be given by

$$
G(x)=\int_{b}^{x} k(t) d t \quad \text { for } \quad b \geq a,
$$

where $t \mapsto k(t)$ is a monotone function. Then for $\sigma \geq 0$ we have that

$$
G(x)=e^{\sigma x} A(x) \quad \text { implies } \quad \frac{k(x)}{G(x)} \rightarrow \sigma \quad(\text { as } x \rightarrow \infty),
$$

where $A(x)$ is an arbitrary translational slowly varying function. (For proof of this statement see: Tasković [29].)

\section{References}

[1] J. Aczél: Functional Equations and Their Applications, Academic Press, New York, 1966.

[2] J. M. Ash, P. Erdös, and L.A. Rubel: Very slowly varying functions, Aequationes Math., 10(1974), 1-9.

[3] V. G. Avakumović: On a O-inverse theorem (in Serbian), Rad Jugoslovenske Akademije Znanosti i Umjetnosti, t. 254 (Razreda MatematičkoPrirodoslovnoga 79) (1936), 167-186.

[4] S. Aljančić, and D. Aranđelović: $\mathcal{O}$-regularly varying functions, Publ. Inst. Math. (Beograd), 22 (1977), 5-22.

[5] N. K. Bari, and S. B. Stečkin: Best approximation and differential properties of two conjugate functions (in Russian), Trudy Mosk. Mat. Obšč., 5(1956), 483-522.

[6] H. Baummann: Umkehrsätze für das asymptotische Verhalten linearer Folgentransformationen, Math. Z., 98 (1967), 140-178.= Théoremes d'inversion relatifs au comportement asymptotique des transformations des suites. Thèse Université de Genève.

[7] N. H. Bingham, C.M. Goldie, and J.L. Teugels: Regular Variation, Cambridge Univ. Press, Cambridge, 1987.

[8] R. Bojanić, and E. Seneta: Slowly Varying Functions and Asymptotic Relations, J. Math. Anal. Appl., 34(1971), 302-315.

[9] R. Bojanić, and J. Karamata: On slowly varying functions and asymptotic relations, Math. Res. Centre Tech. Summary Report 432. Madison: University of Wisconsin 1963. 
[10] R. Bojanić, and E. Seneta: A unified theory of regularly varying sequences, Math. Zeitschrift, 134 (1973), 91-106.

[11] N. G. de Bruijn: Pairs of slowly oscillating functions occuring in asymptotic problems concerning the Laplace transform, Nieuw Arch.Wisk., 7(1959), 20-26.

[12] J. Galambos, and E. Seneta: Regularly varying sequences, Proc. Amer. Math. Soc., 41 (1973), 110-116.

[13] H. Delange: Sur un théorème de Karamata, Bull.Sci.Math. France, 79(1955), $9-12$.

[14] L. de Haan: On regular Variation and Its Application to the Weak Convergence of Sample Extremes, Mathematical Centre Tracts 32, Amsterdam, 1970.

[15] G.H. Hardy, and J.E. Littlewood: Notes on the theory of series XI-On Tauberian theorems, Proc. London Math. Soc., 30 (1929), 23-37.

[16] J. Karamata: Sur certains "Tauberian theorems" de M.M. Hardy et Littlewood, Mathematica (Cluj), 3 (1930), 33-48.

[17] J. Karamata: Sur un mode de croissance régulière des fonctions, Mathematica (Cluj), 4(1930), 38-53.

[18] J. Karamata: Sur un mode de croissance régulière, Théorèmes fondamentaux, Bull.Soc.Math. France, 61(1933), 55-62.

[19] J. Karamata: Bemerkung über die vorstehende Arbeit des Herrn Avakumović mit, näherer Betrachtung einer Klasse von Funktionen, welche bei den Inversionssätzen vorkommen, Bull. International, de l'Académie Yougoslave, Zagreb, 29 et 30 (1935), 117-123. (This is a contracted version of: A remark of the foregoing paper of Mr V. Avakumovic (in Serbian), Rad Jugoslovenske Akademije znanosti i umjetnosti, t. 254 (Razreda MatematičkoPrirodoslovnoga 79) (1936), 187- 200.)

[20] J.F.C. Kingman: Ergodic properties of continuous-time Markov processes and their discrete skeletons, Proc. London Math. Soc., III. ser. 13 (1963), 593-604.

[21] J. Lamperti: Limiting distributions for branching processes, In: Proc. 5th Berkeley Symp. on Math. Stat. and Prob., Vol. II, Part 2, pp. 225-241 (ed. L. le Cam and J. Neyman). Berkeley and Los Angeles: University of California Press (1967).

[22] W. Matuszewska: Regularly increasing functions in connection with the theory of $L^{* \varphi}$-spaces, Studia Math., 21(1962), 317-344.

[23] W. Matuszewska, and W. Orlicz: On some classes of functions with regard to their orders of growth, Studia Math., 26(1965), 11-24.

[24] G. Pólya: Bemerkungen über unendliche Folgen und ganze Funktionen, Math. Ann., 88 (1923), 169-183. 
[25] R. Schmidt: Über divergente Folgen und lineare Mittelbildungen, Math. Zeitschrift, 22 (1925), 89-152.

[26] E. Seneta: Regularly Varying Functions, Lecture Notes in Mathematics 508, Springer - Verlag, Berlin - Heidelberg - New York, 1976.

[27] R.S. Slack: Further notes on branching processes with mean 1, Z. Wahrscheinlichkeitstheorie verw. Gebiete, 25 (1973), 31-38.

[28] I. Schur: Zur Theorie der Cesaroschen und Hölderschen Mittelwerte, Math. Z., 31 (1930), 391-407.

[29] M. R. Tasković: Translational regularly varying functions, 2001, p. 8, (to appear).

[30] M. R. Tasković: Translational $\mathcal{O}$-regularly varying functions, 2001, p. 27, (to appear).

[31] T. van Aardenne - Ehrenfest, N. G. de Bruijn, and J. Korevaar: A note on slowly oscillating functions, Nieuw Arch.Wisk., 23(1949), 77-86.

[32] D. Đurčić: $\mathcal{O}$-Regularly Varying Functions and Strong Asymptotic Equivalence, J. Math. Anal. Appl., 220 (1998), 451-461.

[33] M. Vuilleumier: Sur le comportement asymptotique des transformations linéaires des suites, Math. Zeitschrift, 98 (1967), 126-139.

[34] A. Zygmund: Trigonometric Series, Vol. 1. Cambridge: University Press (1968).

Matematički fakultet 11000 Beograd, P.O. Box 550

Serbia \& Montenegro

Received February 1, 2001. 